ReVISTA INTERnaCIONAL de SOCIOLOGÍa (RIS)

Vol.68, No 3, SEPTIEMBRE-DICIEMBRE, 603-635, 2010

ISSN: 0034-9712

eISSN: 1988-429X

DOI:10.3989/ris.2008.12.17

\title{
ESTRATEGIAS PARA AUMENTAR LA TASA DE RESPUESTA Y LOS RESULTADOS DE LA ENCUESTA SOCIAL EUROPEA EN ESPAÑA
}

\section{STRATEGIES TO INCREASE THE RESPONSE RATE AND RESULTS OF THE EUROPEAN SOCIAL SURVEY IN SPAIN}

\author{
CLARA RIBA \\ Universitat Pompeu Fabra. Barcelona. España \\ clara.riba@upf.edu \\ MARIANO TORCAL \\ Universitat Pompeu Fabra. Barcelona. España \\ mariano.torcal@upf.edu \\ LaURa Morales \\ Universidad de Manchester. Reino Unido \\ laura.morales@manchester.ac.uk
}

\section{RESUMEN}

El análisis de los datos de la tercera ola de la Encuesta Social Europea en España y su comparación con los de las dos olas precedentes permiten descubrir los factores que más han incidido en la mejora de los resultados y cuál es el perfil de las personas sobre las que es más rentable concentrar los esfuerzos de localización y de conversión de negativas, siendo las localizaciones y las conversiones las que, en definitiva, han constituido las principales claves del éxito. El trabajo muestra que el aumento de la tasa de respuesta de la tercera ola con respecto de las dos olas precedentes fue debido esencialmente a un mayor y mejor control de los procesos de campo, a un incremento de los incentivos a los entrevistadores y a un mayor esfuerzo de localización y conversión de negativas en horarios fuera de la jornada laboral habitual.

\section{Palabras Clave}

Conversión de negativas, Encuesta, Localización, Trabajo de campo.

\begin{abstract}
The longitudinal analysis of the contact form data of the third round of the European Social Survey in Spain allows us to identify the most successful strategies in order to increase response rates in social and political surveys in Spain. The analysis of these data provides us with very useful information to estimate what are the likely gains in response rate due to the improvements of fieldwork design and supervision. More especially, we show in this paper that concentrating fieldwork efforts in locating and converting refusals with specific strategies such as a better controlling of interviewers, increasing incentives, and targeting respondents after the regular working hours and during weekends really pay off.
\end{abstract}

\section{KEYWORDS}

Fieldwork, Locating, Refusal Conversion, Survey. 


\section{INTRODUCCIÓN ${ }^{1}$}

Los expertos en estudios de encuesta y las empresas encuestadoras conocen sobradamente la dificultad que entraña la realización del trabajo de campo de una encuesta de opinión si se persigue la obtención de datos de calidad. La teoría estadística establece que la mejor manera de obtener una muestra representativa es aplicando los principios de aleatoriedad en todas las etapas del muestreo (Kish 1965; Sudnan 1983) y, una vez seleccionada la muestra, realizando las entrevistas sin aplicar sustituciones y obteniendo una tasa de respuesta tan elevada como sea posible (Bradburn 1985). Estos tres elementos -aleatoriedad, no-sustitución de casos y elevada tasa de respuesta-, son condiciones necesarias, aunque no suficientes, para obtener datos de calidad (Marker 2002). Solamente si se siguen escrupulosamente estos criterios se tienen garantías de que la muestra finalmente obtenida sea una buena representación de la población (Fowler 2002; Stoop 2005).

Sin embargo, la dificultad de localización de algunos de los individuos seleccionados, así como el rechazo a participar en la encuesta por parte de algunos otros, tienen como consecuencia en muchas ocasiones una bajísima tasa de respuesta. Tan baja que, con el objetivo final de obtener un número determinado de entrevistas completadas, generalmente se aplican sustituciones de los individuos no localizados o de aquellos que rehúsan ser entrevistados. Pero, si bien es cierto que una baja tasa de respuesta introduce serias posibilidades de obtener una muestra sesgada, también lo es que la aplicación sistemática de sustituciones no soluciona ese problema (Bethlehem 2002; Stoop 2005). En efecto, si la tasa de respuesta es baja porque hay dificultades de localización o porque hay un elevado número de rechazos, solamente habremos incluido en la muestra real a aquellos individuos de fácil localización y que, además, están bien predispuestos a dar su opinión. Es decir, el estudio ignorará por completo las opiniones de aquellos individuos que están muy ocupados, que tienen jornadas laborales poco habituales o que son reacios a exponer sus ideas. El resultado será una muestra sesgada que no proporciona las opiniones del conjunto de la población sino sólo de una parte de ella (Groves 1989). Pero si, con el objetivo de aumentar la tasa de respuesta, sustituimos a estos individuos por otros, el resultado no mejora. Aunque las sustituciones se hayan seleccionado aleatoriamente, los entrevistadores se encontrarán con el mismo problema: sólo podrán obtener respuestas de aquellos fácilmente localizables y dispuestos a participar (Philippens y Billiet 2004).

En las siguientes páginas queremos mostrar el efecto que un buen diseño de trabajo de campo y un exhaustivo mecanismo de incentivos pueden tener en la mejora de las tasas de respuesta de una encuesta, sin necesidad de emplear sustituciones. En concreto va a evidenciarse, con datos del llamado formulario de contacto de la tercera ola de la European Social Survey (en castellano, Encuesta Social Europea, ESE) en

\footnotetext{
${ }^{1}$ Este trabajo ha sido financiado con las ayudas de los proyectos SEJ2004-21978E, SEJ2006-10974 y SEJ2007-2956E.
}

RIS, VOL.68. № 3, SEPTIEMBRE-DICIEMBRE, 603-635, 2010. ISSN: 0034-9712. DOI: 10.3989/ris.2008.12.17 
España, los factores que más pueden incidir en la mejora de los resultados de la tasa de respuesta de una encuesta, y el perfil de las personas sobre las que es más rentable concentrar los esfuerzos de localización y conversión de negativas para poder lograrlo. Como ya sabemos, las localizaciones y las conversiones son, en definitiva, las que constituyen la clave del éxito para lograr dicho objetivo (Stoop 2005).

El trabajo se estructura en cuatro partes. Las dos primeras discuten respectivamente algunas características técnicas de la ESE relacionadas con la naturaleza del trabajo de campo y el llamado formulario de contacto, así como los factores que han incidido en la mejora de resultados de la tercera ola respecto de los obtenidos en las dos olas anteriores, haciendo especial hincapié en el control establecido, en los incentivos para los entrevistadores y en el aumento de los intentos de contacto fuera de las horas de la jornada laboral habitual. La tercera parte presenta una tipología de ciudadanos definida a partir del resultado del trabajo de campo y describe el perfil de cada grupo en función de los datos recogidos en el formulario de contacto. En la cuarta parte se estiman unos modelos explicativos de la participación en encuestas en España que permiten establecer cuáles son los factores individuales y contextuales que más influyen en los resultados del trabajo de campo. En conjunto, estas cuatro partes cuentan una historia que permite entender los motivos del éxito (en términos comparativos) del trabajo de campo de la tercera ola de la ESE en España.

\section{El diseño MUESTRAL Y LAS CARACTERÍSTICAS DEL tRABAJO dE CAMPO EN LA ESE}

En trabajos anteriores sobre la ESE en España ya se han expuesto en detalle algunos de los aspectos técnicos distintivos de esta encuesta comparada que la convierten en un instrumento único dentro de las ciencias sociales para obtener datos de encuesta de contrastada calidad y rigor científico (Riba y Cuxart 2003; Cuxart y Riba 2005; Torcal, Morales y Riba, 2006).

Por lo que respecta al diseño muestral, estos requisitos establecen lo siguiente: a) la población objeto de estudio está formada por todos los residentes en domicilios particulares, de edad igual o superior a los 15 años, sea cual sea su nacionalidad, ciudadanía 0 lengua; b) la muestra debe ser probabilística en todas sus etapas y debe documentarse la probabilidad de selección de cada una de las unidades de las distintas etapas de muestreo; c) no se admite ningún tipo de sustitución, ni de los casos de rechazo ni de los de no contacto; d) el tamaño muestral efectivo debe ser como mínimo de $1.500^{2}$; y e) la tasa de respuesta mínima debe ser del $70 \%{ }^{3}$.

\footnotetext{
${ }^{2}$ El tamaño efectivo de una muestra es el tamaño de una muestra aleatoria simple con la cual se obtendría el mismo grado de precisión que con la muestra efectivamente seleccionada. Puesto que la ESE establece que en cada país se aplique el diseño muestral más adecuado dadas sus características y los marcos de muestreo disponibles, los diseños muestrales son distintos para cada país y un tamaño efectivo común facilita la comparación entre países.

${ }^{3}$ Las condiciones de aleatoriedad, de no-sustitución y de elevada tasa de respuesta, aunque necesarias, no son suficientes para garantizar unos datos de calidad. Es necesario, también, disponer de un buen cuestionario
} 
En España, en la primera ola de la ESE, se optó por aplicar un muestreo estratificado (Comunidades Autónomas y rural / urbano) polietápico (secciones censales, hogares, individuo). El Instituto Nacional de Estadística (INE) proporcionó la muestra de hogares y el entrevistador era el encargado de seleccionar por un método aleatorio a un individuo dentro de cada hogar. En las olas siguientes, segunda y tercera, se eliminó la segunda etapa puesto que el INE proporcionó directamente una muestra de individuos, con lo que se eliminó una de las fuentes de efecto de diseño, se aumentó el número de estratos mediante una clasificación más fina del tamaño de los municipios y se aumentó la cobertura de la población con la inclusión de Ceuta y Melilla.

Por lo que respecta al trabajo de campo, se establece lo siguiente: a) éste deberá realizarse simultáneamente en todos los países participantes, con una duración mínima de un mes y máxima de cuatro; b) debe garantizarse la formación de todo el personal de campo, con participación directa del coordinador nacional de la ESE en cada país; c) a cada entrevistador podrá encargársele como máximo la consecución de las entrevistas de 48 unidades muestrales; d) la entrevista debe ser cara a cara y el primer contacto con el entrevistado debe ser personal; e) no se puede abandonar una unidad por improductiva hasta que se hayan realizado un mínimo de 4 intentos de contacto, uno de los cuales debe realizarse forzosamente en horas vespertinas y otro en fin de semana; f) se deben elaborar informes quincenales sobre el desarrollo del trabajo de campo que muestren el estado en el que está cada una de las unidades muestrales, la tasa de respuesta conseguida hasta el momento y los controles de calidad realizados ${ }^{4}$. El trabajo de campo en España en la primera ola se ajustó a estas especificaciones, excepto quizás por una escasa participación del coordinador nacional de la ESE y de su equipo en las sesiones formativas, debido a las reticencias de la empresa encuestadora. En las olas posteriores los requisitos del trabajo de campo que se establecieron en España fueron aumentando su exigencia, incluso superándolas, como por ejemplo la elaboración de los informes de trabajo de campo semanales.

y realizar bien el trabajo de campo. Como es bien sabido, la manera como están formuladas las preguntas, las opciones de respuesta disponibles y su estructura influyen también en la calidad de la encuesta. Existe abundante literatura al respecto y se han realizado múltiples experimentos que muestran cómo el resultado a la pregunta sobre un determinado tema varía en función del instrumento de medida utilizado, es decir del ítem 0 pregunta de la encuesta formulada (véase Saris y Munich 1995; Saris et al. 2004; Saris y Gallopher 2007). También se encuentra abundante literatura sobre los sesgos que puede introducir el entrevistador en las respuestas obtenidas en una entrevista cara a cara (Briemer y Lyberg 2003). Características socio-demográficas como el género, la edad o la raza, pero también otros aspectos como la indumentaria, la simpatía o la manera de iniciar la conversación pueden generar confianza o desconfianza en el entrevistado y ser decisivos en la obtención de la entrevista. También la actitud del entrevistador y las respuestas que pueda dar a las dudas de los entrevistados puede dar lugar a sesgos en las respuestas (Briemer y Lyberg 2003).

${ }^{4}$ Tasa de respuesta=número de entrevistas completadas / (número de unidades seleccionadas - número de unidades inelegibles). Las unidades inelegibles son aquellas en las que los individuos seleccionados han muerto, se han mudado a otro país o residen en instituciones, o bien aquellas cuya dirección es errónea por tratarse de una vivienda vacía, todavía no construida o una dirección no residencial. 
La experiencia de las tres primeras olas ha mostrado la dificultad de alcanzar una tasa de respuesta del $70 \%$ puesto que muy pocos países lo han conseguido ${ }^{5}$. Sin embargo, el equipo español de la ESE ha trabajado para mejorar la tasa de respuesta en España y ha llevado a cabo una serie de medidas, conforme a dicho protocolo, obteniendo unos resultados excelentes y pasando de una tasa de respuesta de un 53,2 por ciento en la primera ola y de un 54,9 por ciento en la segunda, a un exitoso 65,9 en la tercera (lo que supone situarse en la posición décima en el ranking de países por tasas de respuesta, frente a las anteriores decimoséptima y decimonovena que se lograron en olas anteriores).

Otro de los aspectos en los que la ESE pone especial énfasis es en la documentación de todo el proceso, estableciendo que para cada unidad de la muestra debe rellenarse un formulario de contacto. Éste recoge todos los intentos de contacto con el individuo, reflejando quién es el encuestador que se ha encargado de cada uno de ellos y cuál ha sido el resultado del intento. El formulario contiene, además, información sobre el entorno físico de la vivienda del entrevistado y una estimación sobre la edad y el sexo del individuo seleccionado para los casos que no finalizan en entrevista. La documentación de todas las incidencias, la monitorización semanal que realiza la propia empresa encuestadora, así como la supervisión de los resultados por parte del equipo español de la ESE proporcionan información importante para el seguimiento detallado y el control de las tareas de campo y posibilitan, en su caso, la toma de decisiones para su mejora durante el periodo de realización de la encuesta.

Es, sin embargo, a posteriori cuando el formulario de contacto se convierte en una herramienta crucial para la evaluación y la mejora de las actividades de campo. La explotación de los datos de las dos primeras olas de la ESE permitió a los responsables de dicha encuesta dos cosas importantes. Por un lado, detectar las debilidades en su estrategia y, por otro, controlar el cumplimiento de las especificaciones de la encuesta. En efecto, la documentación de todas las unidades seleccionadas (y no solamente de las que finalizaron en entrevista) permitió establecer el perfil de los individuos en función de su localización y participación en la encuesta y analizar las características individuales y contextuales que incidieron positivamente en el trabajo de campo (Torcal, Morales y Riba 2006), permitiendo de este modo un rediseño de las estrategias de campo para la tercera ola. Por lo que respecta a las especificaciones de la ESE, posibilitó descubrir incumplimientos de los requisitos por parte de algunos entrevistadores. El análisis de los formularios de contacto de la segunda ola (Torcal, Morales y Riba 2006) puso de manifiesto que las mejoras introducidas en 2004 fueron escasamente efectivas, entre otras causas, porque hubo una deficiente ejecución del trabajo de campo, con el incumplimiento de alguna de las normas para los intentos de contacto establecidas con carácter general por la ESE y de otras específicas establecidas por el equipo español. $25(20 \%)$.

${ }^{5}$ En la primera ola sólo fueron 5 países de $25(23 \%)$, en la segunda 6 de $26(23 \%)$ y en la tercera 5 de 
Como consecuencia, en la tercera ola los esfuerzos se centraron en un mayor control del trabajo de campo, mejoras en los incentivos para los entrevistadores, y una amplitud del esfuerzo de contacto y reconversión de las negativas, con lo que se consiguió una mejora notable de los resultados. Los datos de la tercera ola que aquí analizamos nos van a permitir evaluar si las mejoras sugeridas a raíz de dicho análisis propiciaron, al menos en parte, la espectacular mejora en la tasa de respuesta de la tercera ola de la ESE en España que antes se ha comentado.

\section{FACTORES QUE HAN INCIDIDO EN LA MEJORA DE RESULTADOS DE LA TERCERA OLA DE LA ESE EN EsPaÑa}

Las características del trabajo de campo de la ESE en España han ido evolucionando a lo largo de las tres olas para obtener una mayor efectividad. Dichas características están resumidas en la tabla 1 de forma que se facilita la comparación entre ellas. Se puede observar fácilmente que hubo notables mejoras en la segunda ola con respecto a la primera en casi todos los aspectos, tanto por lo que se refiere al sistema de administración del cuestionario con el uso de CAPI (Computer Assisted Personal Interviewing) como a una mejor formación de los entrevistadores, a una más amplia información para los ciudadanos, al establecimiento de mejores incentivos para entrevistadores y entrevistados y al incremento de los intentos de contacto. Sin embargo, pese a todo ese esfuerzo, los resultados no fueron muy satisfactorios: si bien es cierto que hubo un aumento de la tasa de respuesta entre la primera y segunda ola (55\% en 2004 frente al 53\% en 2002), ese aumento fue pequeño y situaba los resultados en España muy lejos del objetivo del $70 \%$ establecido para la ESE.

Uno de los cambios de la tercera ola con respecto de la segunda consistió en invertir un mayor esfuerzo en la formación de los entrevistadores y los coordinadores de zona, con sesiones en Madrid y Barcelona, con mejores materiales y con especial énfasis en las estrategias de conversión de negativas. Además, el envío de las cartas de presentación a los entrevistados se organizó de manera secuencial, de tal forma que tras una primera carta de presentación del estudio dos meses antes del inicio de campo se mandaba una segunda carta, junto con un folleto informativo dos semanas antes del primer intento de contacto. De esta forma, se maximizaba la probabilidad de que el individuo seleccionado recordara la información en el momento de recibir el primer contacto y estuviera más predispuesto a la participación ${ }^{6}$.

Por lo que respecta a los incentivos, se mejoraron sustancialmente las retribuciones económicas a los entrevistadores dirigidas principalmente a la consecución del

\footnotetext{
${ }^{6}$ Según los datos españoles de la segunda ola, las tasas de rechazo fueron menores entre los ciudadanos que recordaban haber recibido la carta informativa que entre quienes afirmaban no haberla recibido o no estaban seguros.
} 


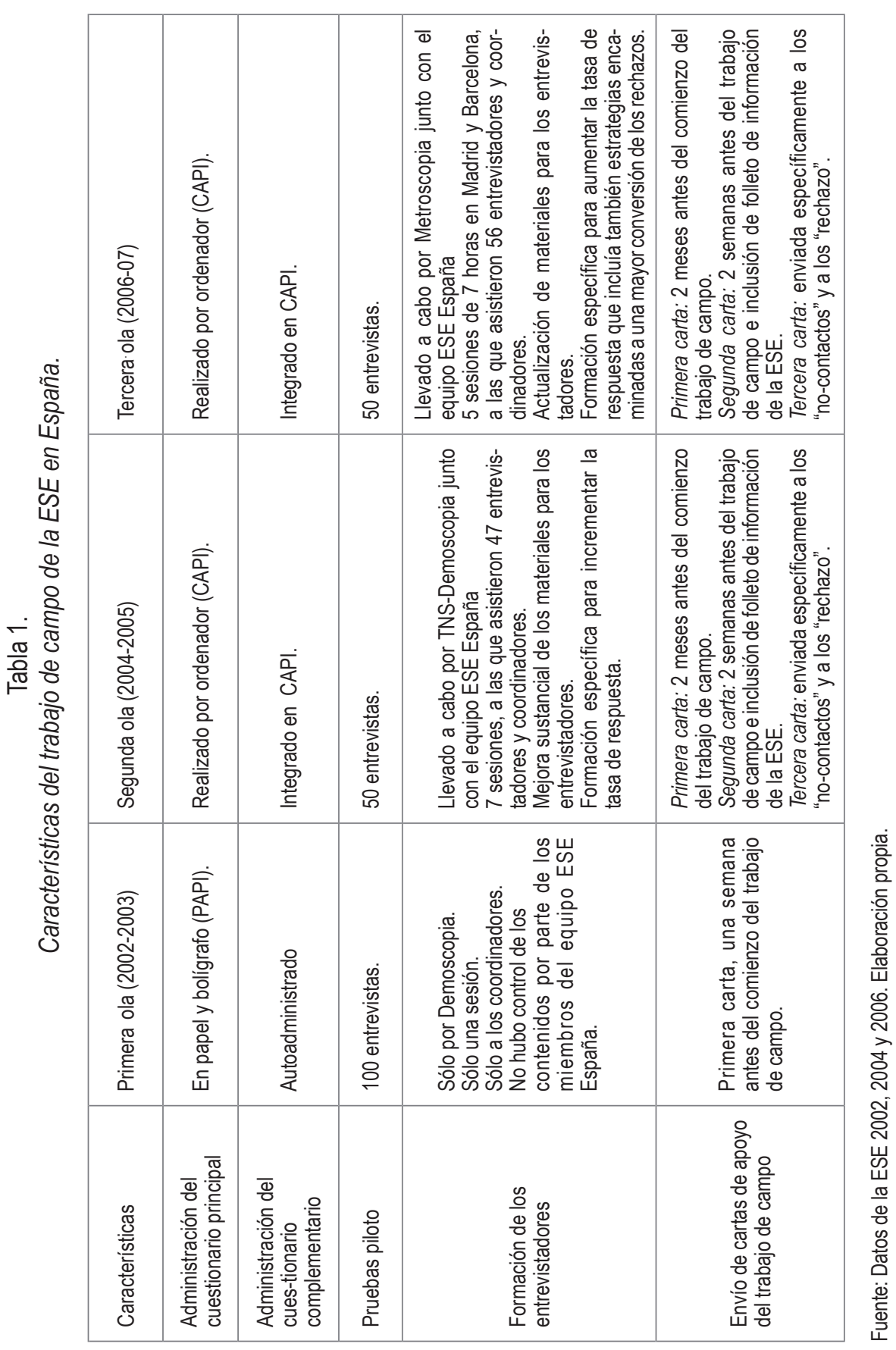

RIS, VOL.68. № 3, SEPTIEMBRE-DICIEMBRE, 603-635, 2010. ISSN: 0034-9712. DOI: 10.3989/ris.2008.12.17 


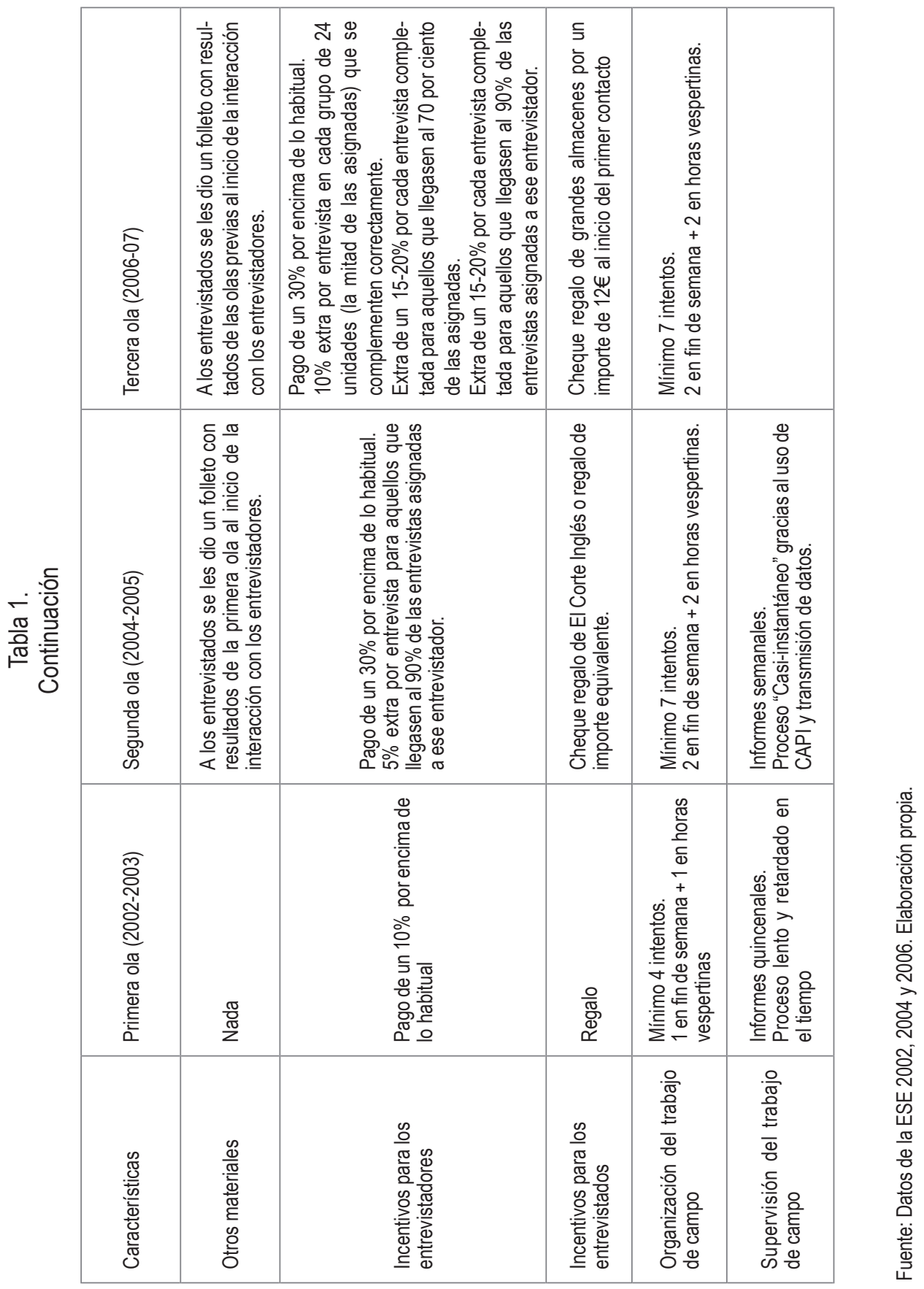

RIS, VOL.68. № 3, SEPTIEMBRE-DICIEMBRE, 603-635, 2010. ISSN: 0034-9712. DOI: 10.3989/ris.2008.12.17 
máximo número de entrevistas (con una bonificación para los entrevistadores que consiguiesen una tasa de respuesta del $70 \%$ y otra bonificación adicional para los que llegasen al 90\%), pero dirigidas también a la correcta documentación de todo el proceso (con otra bonificación por cada grupo de casos con los formularios de contacto correctamente completados). Además, se introdujeron también medidas dirigidas a conseguir una mayor implicación de la empresa que realizó el trabajo de campo, consistentes en una serie de penalizaciones por incumplimiento de las condiciones pactadas en el contrato.

El equipo español mantuvo en la tercera ola las normas sobre la organización del trabajo de campo que había establecido para la segunda y que representaban un esfuerzo adicional al requerido para el cumplimiento de las establecidas por la ESE. Es decir, se debían realizar un mínimo de siete intentos de contacto antes de abandonar una unidad por improductiva (la ESE establece un mínimo de cuatro), de los cuales un mínimo de dos debían ser realizados en horas vespertinas y un mínimo de dos en fin de semana (la ESE establece un mínimo de uno y uno respectivamente). Pero en esta ocasión, a diferencia de lo que ocurrió en la segunda ola, tanto la empresa encuestadora como el equipo español de la ESE intensificaron su control sobre el proceso, lo que redundó en un mejor cumplimiento de las especificaciones establecidas. La tabla 2 muestra la distribución porcentual del número de visitas que recibió cada unidad de la muestra en las tres ediciones de la encuesta.

En los datos de 2006 se observa una disminución del porcentaje de individuos con un único intento de contacto y un aumento considerable de las unidades con tres o más intentos. Este mayor esfuerzo por conseguir contactar con la persona seleccionada para formar parte de la muestra queda también reflejado en el número medio de intentos de contacto que fue de 3,38 por unidad en 2006 cuando había sido sólo de 2,58 en 2004.

Parece, pues, claro que en 2006 se realizó un esfuerzo suplementario en los intentos de contacto en comparación con las dos olas precedentes. Pero no sirve de mucho intensificar los intentos de contacto si estos se repiten en el mismo horario en el que han resultado ser infructuosos. Por lo tanto, también es importante saber si hubo también una mejora en el cumplimiento de los requisitos relativos a las horas en las que se realizaron estos intentos.

Los datos de la tabla 3 muestran claramente un mejor cumplimiento de las especificaciones de campo en la tercera ola que en la segunda ya que hubo una reducción importante en el número de unidades muestrales que no recibieron intentos de contacto en horario vespertino o en fin de semana. Ciertamente, sigue habiendo porcentajes elevados de individuos sin intentos de contacto en esas franjas horarias, pero ello no necesariamente constituye un incumplimiento, puesto que puede tratarse de individuos que fueron contactados en los primeros intentos realizados en horario laboral sin que, por consiguiente, fuera necesario intentarlo en otros momentos. Para conocer los incumplimientos el análisis se centra, pues, en aquellas unidades muestrales que requirieron más de cuatro visitas del entrevistador. Para esas unidades, alguna de las visitas sí que debía ser realizada necesariamente en horario vespertino y otra en fin de semana. $E$ 
Tabla 2.

Número de visitas que recibió la unidad.

(Porcentajes de unidades sobre el total de la muestra)

\begin{tabular}{lrrr}
\hline & 2002 & 2004 & 2006 \\
\hline 0 & 1,5 & 0,0 & 0,0 \\
1 & 36,9 & 38,0 & 18,7 \\
2 & 20,6 & 24,9 & 24,5 \\
3 & 13,2 & 15,4 & 18,0 \\
4 & 9,4 & 8,0 & 13,9 \\
5 & 9,5 & 4,6 & 9,8 \\
6 & 4,2 & 3,3 & 6,6 \\
7 & 2,9 & 2,2 & 3,7 \\
8 & 1,2 & 1,6 & 1,7 \\
9 & 0,6 & 0,7 & 1,2 \\
10 o más & 0,2 & 1,2 & 2,2 \\
Total & 100,0 & 100,0 & 100,0 \\
N & 3.702 & 3.213 & 3.290 \\
Mínimo & 0 & 1 & 1 \\
Máximo & 10 & 37 & 17 \\
Media & 2,64 & 2,58 & 3,38 \\
Desviación Típica & 1,89 & 2,10 & 2,21 \\
\hline E & & &
\end{tabular}

Fuente: Datos de la ESE 2002, 2004 y 2006. Elaboración propia.

Tabla 3.

Porcentaje de hogares / individuos que nunca recibieron un intento de contacto en...

\begin{tabular}{lccc} 
& 2002 & 2004 & 2006 \\
\hline Tarde (5-7) & 51 & 50 & 42 \\
Noche (8-12) & 75 & 77 & 66 \\
Tarde-noche (7-12) & 62 & 64 & 52 \\
Durante el fin de semana & 66 & 75 & 60 \\
\hline
\end{tabular}

Fuente: Datos de la ESE 2002, 2004 y 2006. Elaboración propia.

RIS, VOL.68. № 3, SEPTIEMBRE-DICIEMBRE, 603-635, 2010. ISSN: 0034-9712. DOI: 10.3989/ris.2008.12.17 
gráfico 1 y el gráfico 2 muestran esa información. La interpretación de los mismos es clara. Para ese grupo de unidades "difíciles" por tratarse de ciudadanos que no pudieron ser entrevistados en los cuatro intentos anteriores, en la encuesta del 2006 aumentaron espectacularmente las visitas en fin de semana y aumentaron también, aunque en menor proporción, las visitas a partir de las 7 de la tarde. Y este dato es mucho más relevante si se tiene en cuenta que también aumentó espectacularmente el porcentaje de unidades que recibieron más de cuatro visitas: fueron un $24 \%$ en 2006 frente a un $14 \%$ en 2004.

La reasignación de unidades a un segundo entrevistador está aconsejada cuando el individuo a entrevistar se ha mudado de domicilio y éste se halla fuera del radio de acción del primer entrevistador o cuando el primer entrevistador ha realizado cuatro intentos de contacto sin éxito. Además, otra de las estrategias recomendadas por la ESE consiste en intentar reconvertir las negativas "suaves" pero persistentes a participar en el estudio mediante la reasignación del caso a un entrevistador distinto al que ha realizado los primeros contactos. La idea es que un segundo entrevistador más experimentado puede que sea más persuasivo que el primero y que obtenga la entrevista donde el primero ha fracasado.

La tabla 4 muestra la distribución de las unidades muestrales de cada una de las tres olas de la ESE en función del número de entrevistadores que intervinieron en cada caso. Una ojeada a la misma nos muestra la disminución importante de los casos asignados a un único entrevistador. Además nos informa de un aumento de la asignación a un segundo con respecto a la segunda ola y a un tercero con respecto a la primera.

¿Se trata de un buen o un mal síntoma el hecho de que más un tercio de los individuos hayan sido visitados por más de un entrevistador? La respuesta depende de cuáles sean los casos reasignados. Si el segundo entrevistador ha sido asignado

Tabla 4.

Número de entrevistadores que se ocupó de cada unidad (\%)

\begin{tabular}{lrrr}
\hline & 2002 & 2004 & 2006 \\
\hline 1 & 73 & 76 & 65 \\
2 & 26 & 16 & 29 \\
3 & 1 & 8 & 6 \\
Total & 100 & 100 & 100 \\
$N$ & 3.646 & 3.213 & 3.290 \\
\hline
\end{tabular}

Fuente: Datos de la ESE 2002, 2004 y 2006. Elaboración propia. 
Gráfico 1.

Número de visitas en fin de semana para unidades que recibieron más de 4 visitas (\%)

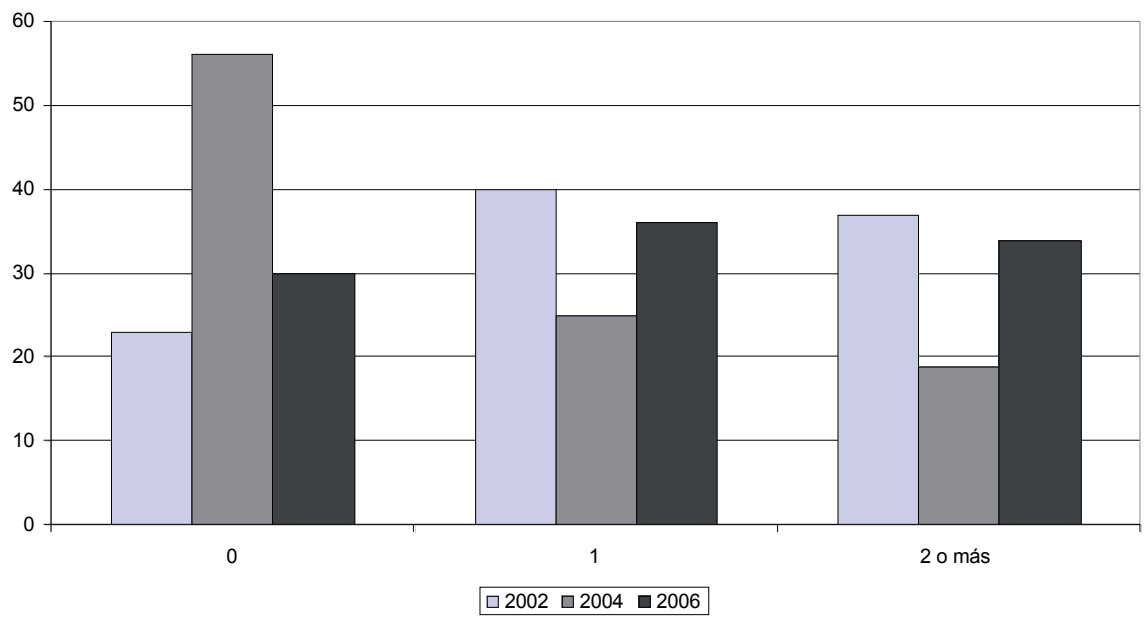

Fuente: Datos de la ESE 2002 y 2006. Elaboración propia

Gráfico 2.

Número de visitas de 7 a 12 de la noche para hogares que recibieron más de 4 visitas

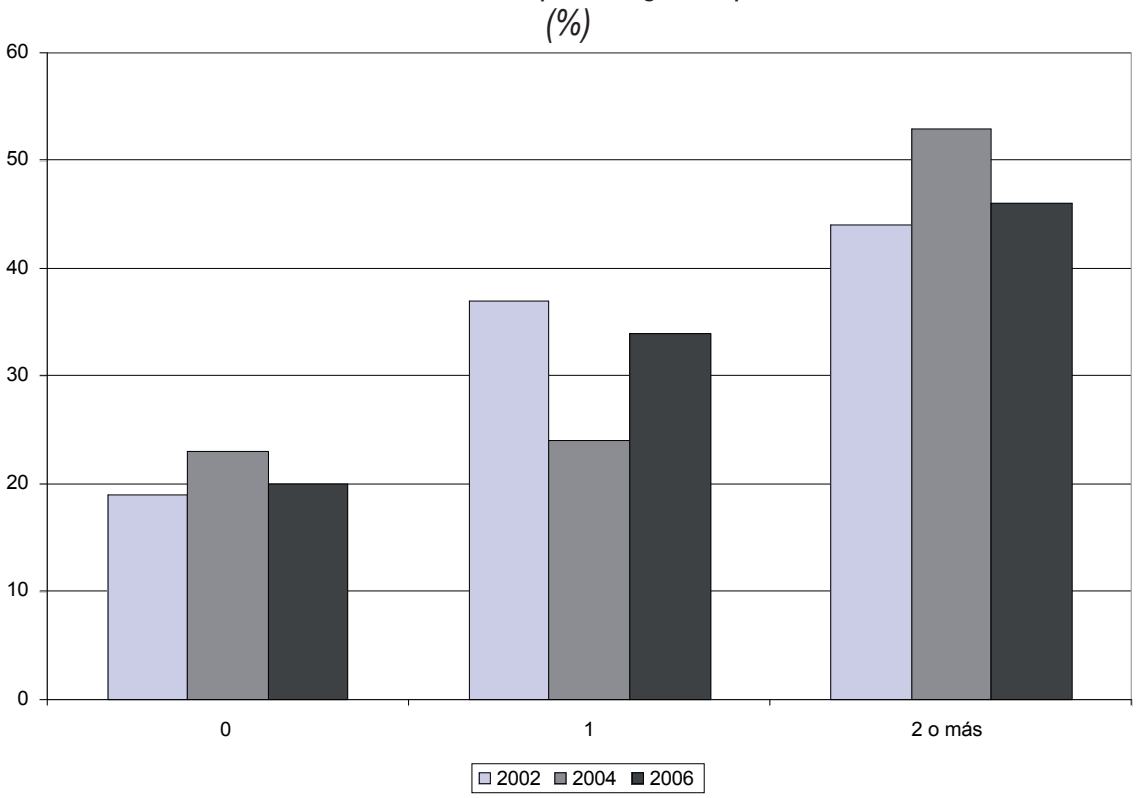

Fuente: Datos de la ESE 2002 y 2006. Elaboración propia

RIS, VOL.68. № 3, SEPTIEMBRE-DICIEMBRE, 603-635, 2010. ISSN: 0034-9712. DOI: 10.3989/ris.2008.12.17 
después de las cuatro primeras visitas se está actuando de acuerdo con el protocolo de la ESS, aumentando los esfuerzos de localización de los entrevistados o de conversión de negativas. Si por el contrario, el segundo encuestador se asigna en las etapas iniciales del proceso probablemente la reasignación sea debida al abandono del primer encuestador. Y ello no es positivo, ya que una elevada tasa de abandono de los entrevistadores requiere de la incorporación de nuevo personal que no ha asistido a las sesiones de briefing, en detrimento de su formación, obligando a reiniciar el trabajo desde el inicio y redundando todo ello en una menor efectividad de las visitas. La tabla 5 muestra la distribución de las unidades muestrales que han recibido más de cuatro visitas en función del número de entrevistadores que han participado en ellas. Los datos muestran que casi la mitad (un 44\%) de las unidades que recibieron más de cuatro visitas fueron reasignadas a un segundo entrevistador en 2006, lo cual cumple con lo establecido en el protocolo y representa un aumento importante respecto a la ola del 2004. Y, por otra parte, el hecho de que para ese grupo difícil haya una disminución de unidades que fueron reasignadas a un tercer entrevistador (actuación no prevista por la ESE), significa que ha disminuido el factor negativo derivado de una excesiva rotación de entrevistadores que había sido frecuente en la segunda ola.

Para finalizar este apartado sobre los esfuerzos de localización de los entrevistados y la reconversión de negativas realizados durante el trabajo de campo, se presentan en la tabla 6 los datos correspondientes a aquellas unidades en las que nunca se localizó a alguien (o en los que en la última visita el individuo no estaba disponible). La misma tabla muestra un descenso muy importante en los incumplimientos que hubo con respecto a la ola precedente, con sólo un $14 \%$ de unidades en las que se incumplieron las tres normas en 2006 frente a un 51\% en 2004.

Tabla 5.

Entrevistadores que se ocuparon de cada unidad que recibió más de 4 visitas (\%)

\begin{tabular}{lrrr}
\hline & 2002 & 2004 & 2006 \\
\hline 1 & 27 & 34 & 40 \\
2 & 68 & 31 & 44 \\
3 & 5 & 35 & 16 \\
Total & 100 & 100 & 100 \\
& 19 & 14 & 24 \\
\hline
\end{tabular}

Fuente: Datos de la ESE 2002, 2004 y 2006. Elaboración propia. 
Tabla 6.

Incumplimiento normas de visitas para unidades no contactadas*

\begin{tabular}{lrrr}
\hline & 2002 & 2004 & 2006 \\
\hline \% que no recibieron 4 visitas & 17 & 76 & 27 \\
\% que no recibieron visita en fin de semana & 22 & 80 & 25 \\
$\%$ que no recibieron visita a partir de las 7 & 25 & 65 & 25 \\
$\%$ que incumplieron las tres normas & 11 & 51 & 14 \\
\hline $\mathrm{N}$ total de unidades afectadas & 378 & 349 & 392 \\
$\%$ unidades afectadas sobre total de la muestra & 10 & 11 & 12 \\
$\%$ unidades afectadas sobre total muestra válida (descontados inelegibles) & 11 & 12 & 14 \\
\hline
\end{tabular}

Fuente: Datos de la ESE 2002, 2004 y 2006. Elaboración propia.

* En 2002 y 2004 se incluyen también las unidades no disponibles en la última visita.

Con el objetivo de poder realizar una evaluación global de la calidad del trabajo de campo de la tercera ola de la ESE en España, en la tabla 7 se presenta el resultado final del mismo comparándolo con las olas precedentes. En ella, todas las unidades muestrales para las que no se ha conseguido obtener una entrevista completa son clasificadas de acuerdo con una detallada tipología establecida por la ESE. Así, se clasifica como "rechazo" a aquellas unidades muestrales en las que ya sea el propio individuo seleccionado o bien la persona con la que se ha contactado en su domicilio no ha accedido a la realización de la entrevista. La categoría de "no contacto" se utiliza para aquellas unidades muestrales en las que el entrevistador no ha conseguido contactar con el individuo seleccionado en ninguno de los intentos realizados. Son clasificadas como "no disponibles" aquellas unidades en las que el individuo seleccionado forma parte de la población pero no ha podido ser entrevistado por estar ausente o enfermo durante el periodo de realización del trabajo de campo o por la existencia de barreras lingüísticas. Finalmente, las "unidades inelegibles" corresponden a individuos que no forman parte de la población objeto de estudio (residen en instituciones, se han mudado a otro país o han muerto) o bien a aquellas cuya dirección es errónea (por ser una vivienda vacía, todavía no construida o una dirección no residencial).

En la tabla 7 destaca la importante reducción en el número de individuos no contactados y no disponibles que se observa en la tercera ola con respecto de la segunda, poniendo de manifiesto una vez más el notable esfuerzo de localización de los casos 
Tabla 7.

Resultado final del trabajo de campo en las tres olas

\begin{tabular}{lcccccc}
\hline & \multicolumn{2}{c}{2002} & \multicolumn{2}{c}{2004} & \multicolumn{2}{c}{2006} \\
\cline { 2 - 6 } Resultado Final & Frecuencia & $\%$ & Frecuencia & $\%$ & Frecuencia & $\%$ \\
\hline Entrevistas completas & 1.736 & 47 & 1.663 & 52 & 1.876 & 57 \\
Rechazo & 1.041 & 28 & 553 & 17 & 559 & 17 \\
No-contacto & 419 & 11 & 360 & 11 & 225 & 7 \\
No disponibles & 161 & 4 & 368 & 11 & 167 & 5 \\
Inelegibles & 286 & 8 & 180 & 6 & 430 & 13 \\
Entrevistas pendientes & 0 & 0 & 89 & 3 & 0 & 0 \\
Otros & 59 & 2 & 0 & 0 & 33 & 1 \\
Total & 3.702 & 100 & 3.213 & 100 & 3.290 & 100 \\
\hline
\end{tabular}

Fuente: Datos de la muestra española de la ESE 2002, 2004 y 2006. Elaboración propia.

incluidos en la muestra realizado en la encuesta del 2006. Probablemente, los resultados habrían podido ser mejores si la muestra no hubiera contenido un porcentaje de individuos inelegibles muy superior al de las olas anteriores (13\%).

Estos resultados deben ponerse en relación con los objetivos marcados por la ESE de obtener una tasa de respuesta del $70 \%$ y un número mínimo de entrevistas equivalente a un tamaño efectivo de 1.500 casos $^{7}$. Habitualmente un tamaño efectivo de 1500 requiere la obtención de un número superior de entrevistas completadas, número que depende del diseño aplicado para la obtención de la muestra en cada caso ${ }^{8}$. La tabla 8 muestra el grado de cumplimiento de estos objetivos en las tres olas y pone de relieve el espectacular salto dado en la tercera.

\footnotetext{
${ }^{7}$ El tamaño efectivo de una muestra es el tamaño de una muestra aleatoria simple que tendría el mismo grado de precisión que la muestra seleccionada. Generalmente las muestras polietápicas tienen un efecto de diseño que debe ser tenido en cuenta en el cálculo de los errores de precisión. Esto, unido a que en el tamaño muestral se estima también la posible tasa de no respuesta, hace que el tamaño efectivo siempre sea menor que el tamaño real de la muestra de la ESE en España.

${ }^{8}$ Véase Cuxart y Riba (2009) sobre el diseño muestral aplicado en la tercera ola de la ESE en España.
} 
Tabla 8.

Grado de cumplimiento de los objetivos de la ESE en España

\begin{tabular}{lccc}
\hline & 2002 & 2004 & 2006 \\
\hline $\begin{array}{l}\text { Número de entrevistas necesarias para alcanzar el } \\
\text { tamaño efectivo de la muestra de 1.500 }\end{array}$ & 2.088 & 1.829 & 1.904 \\
Número de entrevistas completadas & 1.736 & 1.663 & 1.876 \\
Tasa de respuesta & 53,2 & 54,9 & 65,9 \\
Ratio entre las dos primeras magnitudes & 0,83 & 0,91 & 0,98 \\
\hline
\end{tabular}

Fuente: Datos de la muestra española de la ESE 2002, 2004 y 2006. Elaboración propia.

¿Cuáles pueden ser los factores que más han incidido en la mejora de la tasa de respuesta? La tabla 9, que presenta el grado de éxito en los intentos de reconversión de negativas, y la tabla 10, que presenta el grado de éxito en los intentos de localización, nos dan las pistas. En 2006 hubo un mayor número de intentos de conversión de las negativas a la participación que en las olas precedentes y, además, dichos intentos fueron exitosos en casi tres de cada cuatro ocasiones. El incremento en los intentos de conversión refleja el mayor esfuerzo en esa dirección desarrollado en la tercera ola y, probablemente, el incremento en el porcentaje de éxito de esos intentos sea la consecuencia de la aplicación de las estrategias específicas proporcionadas a los entrevistadores en las sesiones iniciales de formación con este objetivo.

Tabla 9.

Grado de éxito en los intentos de conversión

\begin{tabular}{lccc}
\hline & 2002 & 2004 & 2006 \\
\hline $\begin{array}{l}\text { Intentos de conversión } \\
\quad \text { (\% sobre el total de negativas de participación) }\end{array}$ & $9 \%$ & $24 \%$ & $28 \%$ \\
$\quad$ Número de intentos & $(104)$ & $(175)$ & $(212)$ \\
Convertidos (\% sobre el total de intentos de conversión) & $46 \%$ & $45 \%$ & $73 \%$ \\
\hline
\end{tabular}

Fuente: Datos de la muestra española de la ESE 2002, 2004 y 2006. Elaboración propia. 
Por lo que respecta a los esfuerzos de localización de las unidades que no pudieron ser localizadas en los cuatro primeros intentos, también en la tercera ola se observa una mejora en el porcentaje de éxitos. En efecto, más de la mitad de los intentos de localización dieron fruto y terminaron en entrevista, si bien ese éxito de la localización queda matizado por el hecho de haber reducido los intentos.

Tabla 10.

Grado de éxito en los intentos de localización

\begin{tabular}{lccc}
\hline & 2002 & 2004 & 2006 \\
\hline $\begin{array}{l}\text { Intentos de localización más allá de la cuarta visita } \\
\text { (\% sobre el total de no localizados en los } 4 \text { primeros } \\
\text { intentos) }\end{array}$ & 89 & 82 \\
$\begin{array}{l}\text { Localizados y con entrevista completada } \\
\text { (\% sobre el total de intentos de localización más } \\
\text { allá de la cuarta visita) }\end{array}$ & 18 & 35 \\
\end{tabular}

Fuente: Datos de la muestra española de la ESE 2002, 2004 y 2006. Elaboración propia.

Hasta ahora se han analizado las distintas estrategias aplicadas en el trabajo de campo y se ha valorado su posible incidencia en el resultado de manera independiente. A continuación se presenta un modelo de predicción del éxito en las tareas de campo a partir del conjunto de factores mencionados anteriormente. La tabla 11 presenta los resultados de la estimación de un modelo logístico de predicción de la probabilidad de obtener la entrevista completada para cada individuo de la muestra. Los resultados muestran que, tal como se esperaba, tanto en 2004 como en 2006 aquellos individuos que recuerdan haber recibido la carta informativa con anterioridad al primer intento de contacto, tienen una mayor probabilidad de aceptar ser entrevistados. El número total de visitas recibidas por el individuo, el número de entrevistadores involucrados en las mismas y el número de visitas en fin de semana muestran tener efectos significativos en la predicción del éxito, pero su efecto es negativo. Este resultado, aunque aparentemente contraintuitivo, lo único que está reflejando es el mayor esfuerzo de localización y reconversión que se ha dedicado a las unidades especialmente difíciles. Lo que nos muestra la regresión es que ese conjunto de variables están asociadas negativamente con la probabilidad de obtener una entrevista y, por lo tanto, son útiles para la predicción de esa probabilidad. Pero el sentido común nos dice que la dirección de la causalidad debe de ser la inversa. Es decir, cuanto más difícil es obtener la entrevista más visitas se realizan; y cuanto más visitas se realizan, mayores posibilidades hay de que algunas de ellas se realicen en fin de semana y/o que la unidad sea reasignada a un segundo entrevistador. 
Tabla 11.

Regresión logística de predicción del éxito en 2004 y 2006

(Variable dependiente: probabilidad de entrevista).

\begin{tabular}{lrrrr}
\hline & \multicolumn{2}{c}{2004} & \multicolumn{2}{c}{2006} \\
\cline { 2 - 5 } & B & Sig. & B & Sig. \\
\hline N. ${ }^{\circ}$ de visitas que recibió el hogar & $-0,357$ & 0,000 & $-0,451$ & 0,000 \\
$\mathrm{~N} .{ }^{\circ}$ de visitas en fin de semana & $-0,122$ & 0,089 & $-0,065$ & 0,202 \\
$\mathrm{~N} .{ }^{\circ}$ de entrevistadores que recibió el hogar & $-0,754$ & 0,000 & $-1,130$ & 0,000 \\
Recibió carta & 0,443 & 0,000 & 0,544 & 0,000 \\
Constante & 1.982 & 0,000 & 2.540 & 0,000 \\
\hline $\mathrm{N}$ & 2,203 & & 2,558 & \\
Chi $^{2}$ del modelo & 369,04 & 0,000 & 651,76 & 0,000 \\
$\mathrm{R}^{2}$ de Nagelkerke & 0,208 & & 0,300 & \\
\hline
\end{tabular}

Fuente: Datos de la muestra española de la ESE 2002, 2004 y 2006. Elaboración propia.

Nota: La variable dependiente excluye los casos inelegibles.

\section{Perfil de la participación en encuestas en España}

Los datos de la tabla 7 muestran el resultado final del trabajo de campo según la clasificación utilizada por la ESE. Dicha clasificación de las unidades de la muestra sólo tiene en cuenta la situación de cada una de ellas al final de todo el proceso. Cuando se cierra el trabajo de campo, cada unidad es clasificada en la categoría que le corresponde según lo que se haya reflejado en la última entrada del formulario de contacto: entrevista completada, rechazo, no contacto, no disponible o inelegible. Siendo esos números importantes, no lo son todo. Es importante también saber hasta qué punto han sido útiles las estrategias de localización de entrevistados y conversión de los rechazos aplicadas en cada una de las ocasiones y qué tipo de individuos son más fáciles de convencer y de localizar con el objetivo de centrar los esfuerzos allí donde haya mayores posibilidades de éxito. A tal efecto, se ha elaborado una tipología, distinta a la anterior, que contempla cuál ha sido el proceso a través del cual cada una de las unidades de la muestra ha llegado al resultado final. En esa clasificación se han excluido los casos no disponibles y los no elegibles (así como los casos excepcionales que en la segunda ola tenían citas pendientes en el momento del cierre del trabajo de campo). La tipología se ha construido pensando en las posibles alternativas de cada etapa del trabajo de campo y se basa en el siguiente esquema: 
a) Supongamos que un individuo de la muestra es asignado a un entrevistador y éste realiza un primer intento de contacto. En ese primer intento el encuestador puede que establezca contacto con el entrevistado (o con algún familiar) o puede que no lo consiga.

b) En el caso de que se haya podido establecer contacto, el individuo que forma parte de la muestra tiene tres alternativas: acceder a la realización de la entrevista desde el inicio; rehusar a participar en la encuesta y seguir negándose a ser entrevistado en las visitas posteriores; o tener una actitud inicial de rechazo pero dejarse convencer en visitas posteriores y acceder finalmente a ser entrevistado.

c) En el caso de que no se haya podido establecer contacto en la primera visita, el entrevistador realizará otras visitas con posterioridad, visitas que pueden dar lugar también a tres situaciones distintas: el entrevistador consigue localizar al individuo que forma parte de la muestra y éste accede a participar en la encuesta; el entrevistador consigue localizar al individuo pero éste rechaza participar; o bien, el entrevistador no consigue localizar al individuo y no se establece ningún tipo de contacto.

Este esquema da como resultado una tipología de los individuos incluidos en la muestra con las cinco categorías siguientes:

Entrevistas: individuos que realizaron la entrevista y estuvieron de acuerdo en hacerla desde el principio.

Convertidos: individuos que, ellos o sus familiares, rechazaron la encuesta inicialmente pero finalmente decidieron contestar la entrevista.

Localizados: individuos que no pudieron ser contactados inicialmente pero que finalmente sí lo fueron y participaron.

Rechazos: individuos que fueron contactados en la primera o en sucesivas visitas pero que expresamente rechazaron la realización de la entrevista.

No localizados: individuos que nunca pudieron ser contactados ni localizados.

Tabla 12.

Clasificación de las unidades muestrales (\%)*

\begin{tabular}{lrrr}
\hline & 2002 & 2004 & 2006 \\
\hline Entrevista & 50,5 & 49,9 & 46,3 \\
Conversión & 1,5 & 3,0 & 6,3 \\
Rechazo & 32,6 & 21,5 & 20,8 \\
Localizado y participa & 2,3 & 11,6 & 18,3 \\
No localizado & 13,1 & 14,0 & 8,4 \\
Total & 100,0 & 100,0 & 100,0 \\
\hline
\end{tabular}

* Han sido excluidas las unidades inelegibles, no disponibles y con cita pendiente.

Fuente: Datos de la muestra española de la ESE 2002, 2004 y 2006. Elaboración propia. 
La tabla 12 muestra la distribución de las unidades muestrales según esa tipología en las tres olas de la ESE en España. Los datos de esta tabla son muy elocuentes y ponen de manifiesto la amplitud del esfuerzo realizado en el trabajo de campo de la tercera ola de la ESE en España. Respecto del 2004, en 2006 se duplicaron las conversiones de negativas, aumentaron en un $58 \%$ las localizaciones que dieron lugar a entrevista y disminuyeron en un $40 \%$ los casos no localizados. La comparación con los datos de la primera ola proporciona unos datos todavía más espectaculares. En efecto, con respecto del 2002, en 2006 se multiplicaron por 8 las localizaciones, se multiplicaron por 4 las conversiones, y los rechazos y las unidades no localizadas se redujeron en un $36 \%$.

Para el objetivo de seguir mejorando las estrategias de trabajo de campo y conseguir el mayor número posible de entrevistas, es importante conocer el perfil de las personas que pertenecen a cada uno de los grupos de la tipología utilizada. Las preguntas sobre características socio-demográficas de la ESE proporcionan información sobre el perfil de los grupos de individuos que han contestado a la encuesta, es decir, para los que han accedido a realizar la entrevista desde el inicio, para los convertidos y para los localizados. Pero no para aquellos que rechazaron o no fueron localizados. Afortunadamente, también se dispone de cierta información para los que rechazaron participar y los que no fueron localizados, puesto que en el formulario de contacto aparece una estimación de la edad y el sexo del individuo (en el caso en que se haya establecido algún tipo contacto) y las valoraciones del encuestador sobre el estado físico de la vivienda y del entorno en el que vive el individuo seleccionado. La tablas 13, 14 y 15 contienen los perfiles de la participación en encuestas según los datos de la ESE en España de 2002, 2004 y 2006 respectivamente ${ }^{9}$. A continuación se analiza cada una de las características de ese perfil por separado.

Edad. Los individuos convertidos, aquellos que inicialmente rechazan pero posteriormente se dejan convencer y participan, acostumbran a ser de más edad que la media. En efecto, en 2006 la edad media de ese grupo fue de 52 años frente a 46 en el conjunto de la muestra. Si se analiza con más detalle, se observa que entre los convertidos, el grupo de edad que tiene un tamaño significativamente mayor que el del conjunto de la muestra es el de mayores de 60 años, mientras que el grupo que lo tiene significativamente menor es el de jóvenes de entre 20 y 39 años ${ }^{10}$. Por el contrario, puede afirmarse que los localizados acostumbran a ser individuos más jóvenes que la media, siendo la edad media de ese grupo (39 años en 2006) entre cinco y siete años inferior a la del conjunto de la muestra en cada una de las tres olas. Concretamente, entre los localizados, son mayoría los individuos menores de 40 años, probablemente debido a que los mayores de 40 años se localizan en mayor proporción que los jóvenes en los primeros intentos.

\footnotetext{
${ }^{9}$ En la primera ola, las estimaciones del sexo y la edad de los no localizados fueron tan escasas que no han sido incluidas en la tabla por considerar que son escasamente representativas.

${ }^{10}$ El escaso número de individuos convertidos en las dos primeras olas (48 en 2002 y 78 en 2004) imposibilita que se detecten diferencias significativas entre grupos de edad.
} 
Tabla 13.

Perfil de participación en encuesta. ESE en España 2002 (primera ola)

\begin{tabular}{|c|c|c|c|c|c|c|}
\hline & Rechazo & $\begin{array}{c}\text { No } \\
\text { localizado }\end{array}$ & Convertido & Localizado & Entrevista & $\begin{array}{c}\text { Toda } \\
\text { la muestra }\end{array}$ \\
\hline Género: hombres (\%) & $52,6^{*}$ & & 45,8 & 48,6 & 47,3 & 48,7 \\
\hline \multicolumn{7}{|l|}{ Grupos de edad (\%) } \\
\hline Menos de 20 años & $2,0^{*}$ & & 0,0 & 5,5 & 6,0 & 4,9 \\
\hline Entre 20 y 39 años & $26,8^{*}$ & & 33,3 & $45,2^{*}$ & 32,3 & 31,5 \\
\hline Entre 40 y 59 años & $44,4^{*}$ & & 33,3 & 31,5 & $28,0^{*}$ & 32,2 \\
\hline 60 o más años & $26,8^{*}$ & & 33,3 & $17,8^{*}$ & 33,7 & 31,5 \\
\hline \multicolumn{7}{|l|}{ Tipo de domicilio (\%) } \\
\hline Vivienda unifamiliar & 26,6 & 27,8 & 31,3 & 35,1 & 30,5 & 29,0 \\
\hline Piso o apartamento & $72,5^{*}$ & 70,8 & 68,8 & 64,9 & $64,2^{*}$ & 67,9 \\
\hline $\begin{array}{l}\quad \text { Otro } \\
\text { Estado físico de los edificios } \\
\text { de la zona (\%) }\end{array}$ & $0,9^{*}$ & $1,4^{*}$ & 0,0 & 0,0 & $5,3^{*}$ & 3,1 \\
\hline Mal o muy mal estado & $6,4^{*}$ & 9,4 & $2,1^{*}$ & 4,1 & 9,6 & 8,3 \\
\hline Estado satisfactorio & 38,5 & $45,6^{*}$ & 45,8 & $27,4^{*}$ & 40,2 & 40,1 \\
\hline Buen estado & $47,3^{*}$ & $35,7^{*}$ & 41,7 & 43,8 & 41,8 & 42,8 \\
\hline $\begin{array}{l}\quad \text { Muy buen estado } \\
\text { Suciedad y vandalismo } \\
\text { en la zona ( } \%)\end{array}$ & 7,8 & 9,4 & 10,4 & $24,7^{*}$ & 8,4 & 8,7 \\
\hline Nada común & 44,8 & $40,5^{*}$ & 33,3 & 51,4 & 47,3 & 45,5 \\
\hline Poco común & 37,9 & $44,0^{*}$ & 52,1 & 31,1 & 36,2 & 37,9 \\
\hline Bastante común & 14,9 & 13,3 & 14,6 & 16,2 & 13,4 & 14,0 \\
\hline Muy común & 2,4 & 2,2 & 0,0 & 1,4 & 3,1 & 2,7 \\
\hline Edad media (años) & & & 48,9 & $41,7^{*}$ & 48,8 & 48,5 \\
\hline Escolarización media (años) & & & 9,6 & $11,7^{*}$ & 10,2 & 10,2 \\
\hline \multicolumn{7}{|l|}{ Ámbito de residencia (\%) } \\
\hline Gran ciudad o suburbio & & & 43,8 & 33,8 & 25,9 & 30,1 \\
\hline Pequeña ciudad & & & 27,1 & 29,7 & 27,5 & 27,5 \\
\hline Pueblo o granja & & & 29,2 & 36,5 & 43,0 & 42,3 \\
\hline \multicolumn{7}{|l|}{ Nivel de estudios (\%) } \\
\hline Primarios o inferior & & & 39,6 & $25,7^{*}$ & 40,1 & 39,4 \\
\hline Secundarios & & & 43,8 & 47,3 & 46,0 & 46,0 \\
\hline Universitarios & & & 16,7 & $27,0^{*}$ & 13,9 & 14,6 \\
\hline Total casos válidos (\%) & 32,6 & 13,1 & 1,5 & 2,3 & 50,5 & 100,0 \\
\hline N. ${ }^{\circ}$ de casos válidos & 1044 & 419 & 48 & 74 & 1614 & 3199 \\
\hline
\end{tabular}

Porcentajes por columna sobre el total de cada grupo de personas de la muestra y medias.

*Valor que difiere significativamente del valor medio del conjunto de casos documentados para un nivel de significación $\alpha=0,05$.

Fuente: Encuesta Social Europea, 2002. Elaboración propia a partir de los registros del trabajo de campo. 
Finalmente, este resultado concuerda con el del grupo de los rechazos, ya que entre estos son mayoría los individuos de más de 40 años, siendo este patrón consistente a lo largo de las tres olas.

Género. Los datos muestran que en el grupo de localizados el porcentaje de hombres es bastante superior al del conjunto de la muestra (15 y 10 puntos porcentuales en 2004 y 2006 , respectivamente) ${ }^{11}$, mientras que no se observan diferencias significativas entre sexos en el grupo de convertidos. De manera similar a lo que ocurre con la edad, este resultado probablemente sea debido a la mayor facilidad con la que se localizan las mujeres en los primeros intentos.

Tipo de domicilio. Entre los convertidos, los no localizados y los que rechazan hay una mayor proporción de individuos que viven en pisos o apartamentos que la que hay en el conjunto de la muestra. Por su parte, entre los que conceden entrevista, la proporción de individuos que viven en viviendas unifamiliares es mayor que la del conjunto. En el grupo de localizados no se aprecian diferencias significativas respecto del conjunto de la muestra por lo que respecta al tipo de vivienda.

Entorno de la zona de residencia. No se observan patrones claros en esos datos. El único dato que parece ser consistente es que en el grupo de rechazos hay una menor proporción de individuos que viven en zonas con edificios en muy mal estado que la que hay en el conjunto de la muestra.

Nivel de estudios. En el grupo de localizados hay un menor porcentaje de individuos que tienen un bajo nivel de estudios (tienen estudios primarios como máximo) y un mayor porcentaje de individuos con estudios secundarios o universitarios que en el conjunto de la muestra.

Tamaño de hábitat. Los datos de 2006 muestran que en el grupo de convertidos hay mayor porcentaje de individuos que viven en grandes ciudades o suburbios y menor porcentaje de individuos que viven en pequeños pueblos que en el conjunto de la muestra (11 puntos porcentuales de diferencia respecto del conjunto de la muestra en ambos casos). Esas diferencias no se observan ni en 2002 ni en 2004, aunque ello puede ser debido al escaso número de unidades convertidas en esas dos olas. Por su parte, en el grupo de los localizados el porcentaje de individuos que viven en pequeños pueblos es menor que en el conjunto.

Del análisis del perfil de la tipología se extrae que el retrato tipo de los individuos que en un primer momento rechazaron participar en la encuesta pero que posteriormente fueron convencidos y realizaron la entrevista (grupo de convertidos) es:

- Individuos mayores, de 40 años o más, que viven en pisos o apartamentos en grandes ciudades o suburbios.

Por su parte, el retrato tipo de los individuos que no fueron localizados en el primer contacto pero sí lo fueron posteriormente y participaron en la encuesta (grupo de localizados) es:

\footnotetext{
${ }^{11}$ El escaso número de casos de localización no permite detectar diferencias significativas en 2002.
} 
Tabla 14.

Perfil de participación en encuesta. ESE en España 2004 (segunda ola)

\begin{tabular}{|c|c|c|c|c|c|c|}
\hline & Rechazo & $\begin{array}{c}\text { No } \\
\text { localizado }\end{array}$ & Convertido & Localizado & Entrevista & $\begin{array}{l}\text { Toda la } \\
\text { muestra }\end{array}$ \\
\hline Género: hombres (\%) & 45,1 & $31,9^{*}$ & 44,9 & $63,2^{*}$ & 49,3 & 48,6 \\
\hline \multicolumn{7}{|l|}{ Grupos de edad (\%) } \\
\hline Menos de 20 años & $2,8^{*}$ & 3,2 & 9,0 & $13,1^{*}$ & 8,6 & 8,1 \\
\hline Entre 20 y 39 años & $25,5^{*}$ & $16,1^{*}$ & 28,2 & $44,8^{*}$ & 38,9 & 36,5 \\
\hline Entre 40 y 59 años & $42,1^{*}$ & 41,9 & 32,1 & 30,3 & $28,0^{*}$ & 31,4 \\
\hline 60 o más años & $29,6^{*}$ & $38,7^{*}$ & 30,8 & $11,7^{*}$ & 24,4 & 24,1 \\
\hline \multicolumn{7}{|l|}{ Tipo de domicilio (\%) } \\
\hline Vivienda unifamiliar & $33,3^{*}$ & $33,3^{*}$ & $26.9^{*}$ & 37,2 & $43,4^{*}$ & 38,7 \\
\hline Piso o apartamento & $66,0^{*}$ & $65,8^{*}$ & $69,2^{*}$ & 58,1 & $52,4^{*}$ & 58,3 \\
\hline $\begin{array}{l}\text { Otro } \\
\text { Estado físico de los edificios } \\
\text { de la zona (\%) }\end{array}$ & $\mathrm{s}^{0,8^{*}}$ & $0,9^{*}$ & 3,8 & 4,7 & $4,1^{*}$ & 3,0 \\
\hline $\begin{array}{l}\text { Mal o muy mal } \\
\text { estado }\end{array}$ & 3,8 & 4,2 & 0,0 & 5,0 & 4,5 & 4,2 \\
\hline Estado satisfactorio & $26,6^{*}$ & $28,1^{*}$ & $12,8^{*}$ & 20,7 & 20,5 & 22,6 \\
\hline Buen estado & 57,5 & 59,2 & $73,1^{*}$ & 53,2 & 55,3 & 56,6 \\
\hline $\begin{array}{l}\text { Muy buen estado } \\
\text { Suciedad y vandalismo en } \\
\text { la zona (\%) }\end{array}$ & $12,1^{*}$ & $8,6^{*}$ & 14,1 & 21,1 & $19,8^{*}$ & 16,5 \\
\hline Nada común & $40,9^{*}$ & 45,3 & 44,9 & 47,2 & $50,5^{*}$ & 47,2 \\
\hline Poco común & $51,0^{*}$ & 45,8 & 50,0 & 45,2 & $39,0^{*}$ & 43,6 \\
\hline Bastante común & 7,4 & 8,6 & 5,1 & $5,0^{*}$ & 8,9 & 8,0 \\
\hline Muy común & 0,7 & $0,3^{*}$ & 0,0 & $2,7^{*}$ & 1,6 & 1,3 \\
\hline Edad media (años) & & & $48,5^{*}$ & $37,6^{*}$ & 43,9 & 43,0 \\
\hline Escolarización media (años) & & & 11,1 & $12,9^{*}$ & $10,7^{*}$ & 11,2 \\
\hline \multicolumn{7}{|l|}{ Ámbito de residencia (\%) } \\
\hline $\begin{array}{l}\text { Gran ciudad o } \\
\text { suburbio }\end{array}$ & & & 39,7 & 29,4 & 30,1 & 30,4 \\
\hline Pequeña ciudad & & & 20,5 & $33,1^{*}$ & 23,0 & 24,7 \\
\hline Pueblo o granja & & & 39,7 & $37,5^{*}$ & 46,8 & 44,8 \\
\hline \multicolumn{7}{|l|}{ Nivel de estudios (\%) } \\
\hline Primarios o inferior & & & 48,0 & $27,4^{*}$ & 41,0 & 38,9 \\
\hline Secundarios & & & 41,3 & 45,9 & 41,6 & 42,3 \\
\hline Universitarios & & & $10,7^{*}$ & $26,7^{*}$ & 17,4 & 18,8 \\
\hline Total casos válidos (\%) & 21,5 & 14,0 & 3,0 & 11,6 & 49,9 & 100,0 \\
\hline N. ${ }^{\circ}$ de casos válidos & 553 & 360 & 78 & 299 & 1286 & 2576 \\
\hline
\end{tabular}

Porcentajes por columna sobre el total de cada grupo o medias por grupos.

* Valor que difiere significativamente del valor medio del conjunto de casos documentados para un nivel de significación $\alpha=0,05$.

Fuente: Encuesta Social Europea, 2004. Elaboración propia, a partir de los registros del trabajo de campo. 
Tabla 15.

Perfil de participación en encuesta. ESE en España 2006 (tercera ola)

\begin{tabular}{|c|c|c|c|c|c|c|}
\hline & Rechazo & $\begin{array}{c}\text { No } \\
\text { localizado }\end{array}$ & Convertido & Localizado & Entrevista & $\begin{array}{c}\text { Toda la } \\
\text { muestra }\end{array}$ \\
\hline Género: hombres (\%) & 43,5 & 33,3 & 49,7 & $57,1^{*}$ & 43,3 & 47,1 \\
\hline \multicolumn{7}{|l|}{ Grupos de edad (\%) } \\
\hline Menos de 20 años & $1,9^{*}$ & 5,9 & 3,9 & $9,8^{*}$ & 6,3 & 6,1 \\
\hline Entre 20 y 39 años & $29,4^{*}$ & 58,8 & $23,4^{*}$ & $49,9^{*}$ & 32,6 & 35,3 \\
\hline Entre 40 y 59 años & $39,4^{*}$ & 29,4 & 37,0 & $24,9^{*}$ & 32,7 & 32,5 \\
\hline 60 o más años & 29,2 & $5,9^{*}$ & $35,7^{*}$ & $15,3^{*}$ & 28,4 & 26,1 \\
\hline \multicolumn{7}{|l|}{ Tipo de domicilio (\%) } \\
\hline Vivienda unifamiliar & $24,6^{*}$ & $28,1^{*}$ & $23,0^{*}$ & 31,7 & 36,4 & 31,0 \\
\hline Piso o apartamento & $71,1^{*}$ & $76,0^{*}$ & $75,8^{*}$ & 65,0 & $58,1^{*}$ & 64,6 \\
\hline $\begin{array}{l}\quad \text { Otro } \\
\text { Estado físico de los } \\
\text { edificios de la zona (\%) }\end{array}$ & 4,3 & $2,2^{*}$ & $1,2^{*}$ & 3,3 & 5,5 & 4,3 \\
\hline $\begin{array}{l}\text { Mal o muy mal } \\
\text { estado }\end{array}$ & $3,2^{*}$ & 5,8 & 4,3 & 4,3 & 5,9 & 5,0 \\
\hline Estado satisfactorio & 27,5 & 22,9 & 25,9 & 25,0 & 26,8 & 26,2 \\
\hline Buen estado & 50,8 & 52,9 & 53,1 & 55,3 & 51,6 & 52,3 \\
\hline $\begin{array}{l}\quad \text { Muy buen estado } \\
\text { Suciedad y vandalismo en } \\
\text { la zona }(\%)\end{array}$ & 18,4 & 19,1 & 16,7 & 15,4 & 15,6 & 16,5 \\
\hline Nada común & 58,1 & 57,8 & 54,3 & 59,6 & 59,1 & 58,6 \\
\hline Poco común & 35,8 & 31,6 & 34,6 & 30,9 & 34,3 & 33,8 \\
\hline Bastante común & 5,7 & 8,9 & 9,3 & 8,8 & 5,8 & 6,8 \\
\hline Muy común & 0,4 & 1,8 & 1,9 & 0,6 & 0,8 & 0,8 \\
\hline Edad media (años) & & & $51,6^{*}$ & $39,3^{*}$ & $47,3^{*}$ & 45,6 \\
\hline Escolarización media (años) & & & 16,6 & 15,2 & 15,9 & 15,8 \\
\hline \multicolumn{7}{|l|}{ Ámbito de residencia (\%) } \\
\hline $\begin{array}{l}\text { Gran cludad } 0 \\
\text { suburbio }\end{array}$ & & & $38,3^{*}$ & 27,6 & 25,0 & 26,8 \\
\hline Pequeña ciudad & & & 31,2 & 25,8 & 30,3 & 31,8 \\
\hline Pueblo o granja & & & $30,5^{*}$ & $36,6^{*}$ & 44,8 & 41,5 \\
\hline \multicolumn{7}{|l|}{ Nivel de estudios (\%) } \\
\hline Primarios o inferior & & & 45,5 & $24,0^{*}$ & 38,1 & 35,0 \\
\hline Secundarios & & & 41,5 & $56,7^{*}$ & 44,8 & 47,6 \\
\hline Universitarios & & & 13,0 & 19,3 & 17,1 & 17,3 \\
\hline Total casos válidos (\%) & 20,8 & 8,4 & 6,3 & 18,3 & 46,3 & 100,0 \\
\hline N. ${ }^{\circ}$ de casos válidos & 559 & 225 & 169 & 492 & 1248 & 2693 \\
\hline
\end{tabular}

Porcentajes por columna sobre el total de cada grupo o medias por grupos.

*Valor que difiere significativamente del valor medio del conjunto de casos documentados para un nivel de significación $\alpha=0,05$.

Fuente: Encuesta Social Europea, 2006. Elaboración propia, a partir de los registros del trabajo de campo. 
- Individuos jóvenes, de menos de 40 años, hombres, con estudios secundarios 0 universitarios, que viven en ciudades 0 en grandes ciudades 0 suburbios.

\section{Modelos eXPLICATIVOS DE LA PARTICIPACIÓN EN ENCUESTAS}

Al final de la primera parte de este artículo, en la tabla 11, se ha presentado un modelo de predicción de la probabilidad de obtener una encuesta en función de las circunstancias del trabajo de campo que afectaron a cada una de las unidades de la muestra (número de visitas recibidas, número de ellas realizadas fuera de horas laborales, número de entrevistadores involucrados y recuerdo sobre haber recibido una carta informativa). En la segunda parte del trabajo se ha construido una tipología de las unidades muestrales que toma en consideración el proceso mediante el cual se ha llegado al resultado final de la encuesta, y se ha presentado el perfil de los individuos que acaban perteneciendo a cada uno de los cinco grupos: entrevista, conversión, localización, rechazo y no-localización. Lo que se presenta en esta tercera parte es un modelo logístico multinomial de predicción de la pertenencia a cada uno de los grupos de la tipología en función de las características individuales y contextuales de cada unidad de la muestra.

Las tablas 16, 17 y 18 muestran los resultados de la estimación del modelo para los datos del 2002, 2004 y 2006 correspondientes a las tres olas de la ESE en España. En cada tabla se presentan dos modelos distintos separados por una línea vertical. El de la izquierda tiene como variables explicativas las características individuales y contextuales estimadas por el encuestador y reflejadas en el formulario de contacto. Es decir, las estimaciones (o declaraciones de los individuos finalmente encuestados) sobre la edad y el género, así como las percepciones sobre el tipo de vivienda y el entorno físico en el que ésta está ubicada. El modelo de la derecha, en cambio, tiene como variables explicativas, además de las anteriores, otro conjunto de variables, que se ha obtenido directamente a través de las respuestas del individuo a las preguntas de la encuesta, como los años de escolarización, el nivel educativo alcanzado y el tipo de hábitat de residencia. Evidentemente, quedan excluidas de este segundo modelo de regresión todas las unidades que finalizaron sin entrevista (las de los grupos de rechazo y no localizados) por lo que la variable dependiente sólo tiene tres categorías: entrevista, convertido y localizado. En ambos modelos, se toma como categoría de referencia el grupo de los entrevistados ${ }^{12}$. Por lo tanto, los coeficientes significativos indican que la correspondiente variable aumenta o disminuye (según sea el signo) la probabilidad de

${ }^{12}$ En la primera ola de la ESE, el número de unidades no localizadas en las que se cumplimentaron adecuadamente todos los apartados del formulario de contacto de estimación de género y edad y de valoración del entorno físico fue muy escaso. Por ese motivo, la categoría de no localizados ha sido excluida en la primera de las regresiones logísticas multinomiales del 2002. 
Tabla 16.

Modelos explicativos de la participación de los españoles en las encuestas con los datos de la ESE en España 2002 (primera ola).

Modelo logístico multinomial.

Variable dependiente: grupos de individuos según resultado de la encuesta

\begin{tabular}{|c|c|c|c|c|c|}
\hline & \multicolumn{3}{|c|}{$\begin{array}{l}\text { Modelo con } 4 \text { grupos } \\
\text { (categoría de referencia: Entrevista) }\end{array}$} & \multicolumn{2}{|c|}{$\begin{array}{c}\text { Modelo con } 3 \text { grupos } \\
\text { (categoría de referencia: } \\
\text { Entrevista) }\end{array}$} \\
\hline & Rechazo & Convertido & Localizado & Convertido & Localizado \\
\hline Constante & $-1,19^{* *}$ & $-3,70^{* *}$ & $-3,89^{* *}$ & $-2,91^{* *}$ & $-2,51^{* *}$ \\
\hline Género (cat. ref.: hombre) & & & & & \\
\hline $\begin{array}{l}\text { Mujer } \\
\text { Grupos de edad (cat. ref.: } 600 \\
\text { más) }\end{array}$ & $-0,25^{* *}$ & 0,03 & 0,01 & $-0,20$ & \\
\hline Menos de 39 años & $-0,11$ & $-0,15$ & $0,79^{* *}$ & & \\
\hline Entre 40 y 59 años & $0,635^{* *}$ & 0,18 & $0,64^{*}$ & & \\
\hline \multicolumn{6}{|l|}{$\begin{array}{l}\text { Tipo de domicilio (cat. ref.: piso u } \\
\text { otro tipo de vivienda) }\end{array}$} \\
\hline Vivienda unifamiliar & $-0,25^{* *}$ & 0,16 & 0,29 & 0,39 & 0,43 \\
\hline \multicolumn{6}{|l|}{$\begin{array}{l}\text { Estado físico de los edificios de } \\
\text { la zona (cat. ref.: mal, muy mal o } \\
\text { satisfactorio) }\end{array}$} \\
\hline Muy buen estado & 0,17 & 0,53 & 1,41 & 0,49 & $1,27^{\text {t* }}$ \\
\hline Buen estado & $0,23^{* *}$ & 0,13 & $0,49^{*}$ & $-0,02$ & 0,31 \\
\hline \multicolumn{6}{|l|}{$\begin{array}{l}\text { Suciedad y vandalismo en la zona } \\
\text { (cat. ref.: bastante o muy común) }\end{array}$} \\
\hline Nada común & $-0,01$ & $-0,40$ & $-0,34$ & 0,22 & $-0,06$ \\
\hline Poco común & 0,02 & 0,43 & $-0,31$ & $0,86^{*}$ & $-0,04$ \\
\hline Edad & & & & $-0,01$ & $-0,02^{* *}$ \\
\hline $\begin{array}{l}\text { Años de escolarización } \\
\text { Ámbito de residencia (cat. ref.: } \\
\text { pueblo o granja) }\end{array}$ & & & & $-0,07$ & 0,00 \\
\hline Gran ciudad o suburbio & & & & $1,03^{* *}$ & 0,41 \\
\hline Pequeña ciudad & & & & 0,48 & 0,25 \\
\hline \multicolumn{6}{|l|}{$\begin{array}{l}\text { Nivel de estudios (cat. ref.: } \\
\text { universitarios) }\end{array}$} \\
\hline Primarios o inferior & & & & $-0,89$ & $-0,23$ \\
\hline Secundarios & & & & $-0,76$ & $-0,45$ \\
\hline Número de casos válidos & & 2241 & & & 1543 \\
\hline Pseudo R-cuadrado de Nagelkerke & & 0,05 & & & 0,06 \\
\hline Chi-cuadrado del modelo & & $94,75^{\star *}$ & $(G L=24)$ & 40,5 & ${ }^{* *} \quad(G L=24)$ \\
\hline
\end{tabular}

"Coeficiente estadísticamente significativo para un nivel de significación $\alpha=0,10$.

${ }^{* *}$ Coeficiente estadísticamente significativo para un nivel de significación $\alpha=0,05$.

Fuente: Encuesta Social Europea, 2002. Elaboración propia.

RIS, VOL.68. № 3, SEPTIEMBRE-DICIEMBRE, 603-635, 2010. ISSN: 0034-9712. DOI: 10.3989/ris.2008.12.17 
Tabla 17.

Modelos explicativos de la participación de los españoles en las encuestas con los datos de la ESE en España 2004 (segunda ola).

Modelo logístico multinomial.

Variable dependiente: grupos de individuos según resultado de la encuesta

\begin{tabular}{|c|c|c|c|c|c|c|}
\hline & \multicolumn{4}{|c|}{$\begin{array}{l}\text { Modelo con } 5 \text { grupos } \\
\text { (categoría de referencia: Entrevista) }\end{array}$} & \multicolumn{2}{|c|}{$\begin{array}{c}\text { Modelo con } 3 \text { grupos } \\
\text { (categoría de referencia: } \\
\text { Entrevista) }\end{array}$} \\
\hline & Rechazo & $\begin{array}{c}\text { No } \\
\text { localizado }\end{array}$ & Convertido & Localizado & Convertido & Localizado \\
\hline $\begin{array}{l}\text { Constante } \\
\text { Género (cat. ref.: hombre) }\end{array}$ & $-0,12^{* *}$ & $-0,39^{* *}$ & $-3,67^{*+}$ & $-2,47^{* *}$ & $-6,78^{*+}$ & $-2,04^{* *}$ \\
\hline $\begin{array}{l}\quad \text { Mujer } \\
\text { Grupos de edad (cat. ref.: } \\
60 \text { o más) }\end{array}$ & $-0,58^{* *}$ & $1,01^{* *}$ & 0,16 & $-0,58^{* *}$ & 0,20 & $-0,55^{* *}$ \\
\hline Menos de 20 años & $-1,33^{* *}$ & $-1,58$ & $-0,29$ & $1,16^{* *}$ & & \\
\hline Entre 20 y 39 años & $-0,56^{* *}$ & $-1,43^{* *}$ & $0,64^{* *}$ & $0,85^{* *}$ & & \\
\hline $\begin{array}{l}\text { Entre } 40 \text { y } 59 \text { años } \\
\text { Tipo de domicilio (cat. } \\
\text { ref.: piso u otro tipo de } \\
\text { vivienda) }\end{array}$ & $0,23^{*}$ & $-0,15$ & $-0,20$ & $0,79^{\star *}$ & & \\
\hline $\begin{array}{l}\text { Vivienda unifamiliar } \\
\text { Estado físico de los } \\
\text { edificios de la zona (cat. } \\
\text { ref.: mal, muy mal o } \\
\text { satisfactorio) }\end{array}$ & $-0,235^{*}$ & $-1,42^{+*}$ & $-0,73^{* *}$ & $-0,26^{*}$ & $-0,80^{* *}$ & $-0,12$ \\
\hline Muy buen estado & $-0,65^{\star *}$ & $-1,33$ & 0,34 & 0,00 & 0,42 & $-0,17$ \\
\hline $\begin{array}{l}\quad \text { Buen estado } \\
\text { Suciedad y vandalismo en } \\
\text { la zona (cat. ref.: bastante } \\
\text { o muy común) }\end{array}$ & $-0,23^{*}$ & 0,75 & $0,82^{* *}$ & $-0,17$ & $0,94^{* *}$ & $-0,23$ \\
\hline Nada común & 0,25 & 0,18 & 0,77 & $0,70^{* *}$ & 0,70 & $0,52^{*}$ \\
\hline Poco común & 0,35 & $-0,02$ & $0,90^{*}$ & $0,87^{* *}$ & 0,76 & $0,70^{* *}$ \\
\hline Edad & & & & & $0,02^{* *}$ & $-0,01^{* *}$ \\
\hline $\begin{array}{l}\text { Años de escolarización } \\
\text { Ámbito de residencia (cat. } \\
\text { ref.: pueblo o granja) }\end{array}$ & & & & & $0,08^{* *}$ & $0,06^{* *}$ \\
\hline $\begin{array}{l}\text { Gran ciudad o } \\
\text { suburbio }\end{array}$ & & & & & 0,01 & $-0,15$ \\
\hline $\begin{array}{l}\quad \text { Pequeña ciudad } \\
\text { Nivel de estudios (cat. } \\
\text { ref.: universitarios) }\end{array}$ & & & & & $-0,42$ & $0,42^{* *}$ \\
\hline Primarios o inferior & & & & & $1,36^{* *}$ & 0,07 \\
\hline Secundarios & & & & & $1,09^{* *}$ & 0,08 \\
\hline $\begin{array}{l}\text { N. }{ }^{\circ} \text { de casos válidos } \\
\text { Pseudo R-cuadrado de Nag } \\
\text { Chi-cuadrado del modelo }\end{array}$ & kerke & $\begin{array}{r}2,044 \\
0,11 \\
204,36^{* *}\end{array}$ & & $(G L=36)$ & 117, & $\begin{array}{r}1579 \\
0,10 \\
*(G L=24)\end{array}$ \\
\hline
\end{tabular}

"Coeficiente estadísticamente significativo para un nivel de significación $\alpha=0,10$.

"Coeficiente estadísticamente significativo para un nivel de significación $\alpha=0,05$.

Fuente: Encuesta Social Europea, 2004. Elaboración propia. 
pertenencia al grupo indicado con respecto a la probabilidad de aceptar la realización de la encuesta desde el inicio.

Lo primero que debe destacarse es que los dos modelos de cada una de las olas son altamente significativos aunque las medidas de bondad del ajuste no sean muy elevadas. La significación de los coeficientes nos permite comprobar si las relaciones detectadas en el análisis independiente de cada variable se mantienen cuando se controla cada una de ellas por todas las demás en un análisis multivariante.

Los datos muestran que, si las demás circunstancias se mantienen constantes, el ser hombre aumenta la probabilidad de pertenecer al grupo de convertidos y al grupo de localizados, mientras que el ser mujer aumenta la probabilidad de que acceda directamente a la entrevista y disminuye la probabilidad de integrar el grupo de los que rechazan.

De manera consistente, los modelos que usan como variable explicativa la edad declarada por el entrevistado en las tres olas (modelos con tres grupos de la parte derecha de la tabla) indican que con la edad aumenta la probabilidad de formar parte del grupo de los convertidos y disminuye la probabilidad de pertenecer al grupo de los localizados. Los modelos que utilizan también las estimaciones de los encuestadores sobre el grupo de edad al que pertenece cada individuo (modelos con 5 grupos de la parte izquierda de la tabla) indican, también de manera consistente, que con la edad disminuye la probabilidad de integrar el grupo de localizados. Sin embargo, para los convertidos el patrón ya no aparece tan claro. En 2006 los individuos de menos de 40 años tienen, efectivamente, menos probabilidades de formar parte a ese grupo que los de más edad. Pero en 2004 los que más probabilidades tuvieron de ser convertidos fueron los individuos de entre 20 y 39 años, y para 2002 la franja de edad a la que pertenecía el individuo no resultaba significativa.

Los datos no muestran efectos consistentes de la educación a la hora de tratar de explicar la pertenencia a uno u otro grupo de la tipología del resultado de la encuesta. Así, los años de escolarización sólo resultan ser un factor significativo para el grupo de convertidos y de los localizados en la segunda ola, y el disponer de estudios universitarios disminuye más la probabilidad de formar parte del grupo de convertidos que el disponer solamente de estudios primarios o secundarios. Por otra parte, no parece que el nivel de estudios alcanzado influya en las probabilidades de integrar el grupo de localizados.

Por lo que respecta al tamaño de hábitat, los datos de las dos últimas olas muestran que la probabilidad de ser del grupo de localizados es mayor en individuos que viven en pequeñas ciudades ( $\mathrm{y}$, en menor grado, en grandes ciudades) que para los que viven en pueblos y zonas rurales. Por su parte, estar entre los convertidos parece más probable para los que viven en grandes ciudades y en sus conurbaciones urbanas.

Aunque no se observa un efecto consistente para las tres olas, los datos parecen indicar que formar parte del grupo de convertidos y, en menor grado, y de localizados es menos probable para los individuos que viven en casas unifamiliares que para los que habitan en pisos, apartamentos u otros tipos de vivienda. 
Tabla 18

Modelos explicativos de la participación de los españoles en las encuestas con los datos de la ESE en España 2006 (tercera ola).

Modelo logístico multinomial.

Variable dependiente: grupos de individuos según resultado de la encuesta

\begin{tabular}{|c|c|c|c|c|c|c|}
\hline & \multicolumn{4}{|c|}{$\begin{array}{c}\text { Modelo con } 5 \text { grupos } \\
\text { (categoría de ref.: Entrevista) }\end{array}$} & \multicolumn{2}{|c|}{$\begin{array}{c}\text { Modelo con } 3 \text { grupos } \\
\text { (categoría de ref.: Entrevista) }\end{array}$} \\
\hline & Rechazo & $\begin{array}{c}\text { No } \\
\text { localizado }\end{array}$ & Convertido & Localizado & Convertido & Localizado \\
\hline & $-1,07^{* *}$ & $-6,01^{\text {** }}$ & $-1,04^{* *}$ & $-0,98^{* *}$ & $-2,76^{* *}$ & \\
\hline Mujer & $-0,02$ & 0,66 & $-0,29^{*}$ & $-0,48^{* *}$ & $-0,32^{*}$ & $-0,48^{* *}$ \\
\hline Menos de 20 años & $-1,25^{* *}$ & 1,46 & $-0,77^{*}$ & $1,05^{* *}$ & & \\
\hline Entre 20 y 39 años & $-0,22$ & $2,10^{* *}$ & $-0,70^{* *}$ & $0,97^{* *}$ & & \\
\hline Entre 40 y 59 años & $0,09^{*}$ & 1,40 & $-0,20$ & $0,32^{*}$ & & \\
\hline Vivienda unifamiliar & $-0,68^{* *}$ & 0,61 & $-0,76^{* *}$ & $-0,19$ & $-0,57^{* *}$ & $-0,60$ \\
\hline Muy buen estado & 0,19 & 0,91 & $0,48^{*}$ & 0,05 & $0,49^{*}$ & $-0,03$ \\
\hline Buen estado & $-0,09$ & 0,70 & 0,18 & $0,23^{*}$ & 0,19 & 0,14 \\
\hline Nada común & 0,27 & 0,88 & $-0,56^{*}$ & $-0,34$ & $-0,41$ & $-0,25$ \\
\hline Poco común & 0,34 & 0,87 & $-0,62^{* *}$ & $-0,50^{\text {** }}$ & $-0,56^{*}$ & $-0,45^{* *}$ \\
\hline Edad & & & & & $0,01^{* *}$ & $-0,02^{* *}$ \\
\hline Años de escolarización & & & & & 0,00 & $-0,01$ \\
\hline \multicolumn{7}{|l|}{ Ámbito de residencia } \\
\hline \multicolumn{7}{|l|}{ (cat. ref. pueblo o granja) } \\
\hline Gran ciudad o suburbio & & & & & $0,68^{* *}$ & $0,29^{*}$ \\
\hline Pequeña ciudad & & & & & 0,27 & $0,34^{\star *}$ \\
\hline \multicolumn{7}{|l|}{$\begin{array}{l}\text { Nivel de estudios } \\
\text { (cat.ref.: universitarios) }\end{array}$} \\
\hline Primarios o inferior & & & & & $0,54^{*}$ & $-0,16$ \\
\hline Secundarios & & & & & 0,36 & $-0,00$ \\
\hline $\mathrm{N}^{0}$ de casos válidos & & 2293 & & & & 1860 \\
\hline \multicolumn{7}{|l|}{ Pseudo R-cuadrado de } \\
\hline Nagelkerke & & 0,09 & & & & 0,09 \\
\hline Chi-cuadrado del modelo & $200,84^{* *}$ & $(G L=36)$ & & & & $\begin{array}{l}146,96^{* *} \\
(\mathrm{GL}=24)\end{array}$ \\
\hline
\end{tabular}

*Coeficiente estadísticamente significativo para un nivel de significación $\alpha=0,10$.

${ }^{* *}$ Coeficiente estadísticamente significativo para un nivel de significación $\alpha=0,05$.

Fuente: Encuesta Social Europea, 2006. Elaboración propia. 
Tampoco concuerdan los resultados de 2004 y de 2006 en lo que se refiere al efecto del estado de suciedad y vandalismo del entorno físico en el que se halla la vivienda del entrevistado a la hora de explicar la pertenencia al grupo de convertidos y al de los localizados. Mientras que en 2004 la probabilidad fue más alta en ambos casos en las zonas de nula o escasa suciedad y vandalismo que en las zonas más deterioradas, en 2006 ocurrió lo contrario. Una posible explicación para estas discrepancias es la existencia de criterios diversos en la clasificación de estos datos, al tratarse de una variable independiente que depende de la evaluación subjetiva de los entrevistadores.

Finalmente, los datos indican que el buen estado físico del edificio de residencia del encuestado incide positivamente en la probabilidad de que se encuentre entre convertidos y localizados.

La tabla 19 resume los principales resultados de los modelos multivariantes. Estos resultados son muy importantes para la definición de estrategias por parte de los científicos sociales que realizan investigaciones por encuesta y por parte de las empresas que realizan el trabajo de campo de esas encuestas, puesto que les permite diseñar estrategias encaminadas a maximizar la obtención del máximo número de entrevistas completadas mediante la concentración de los esfuerzos de conversión y localización en las unidades que puedan tener mayor probabilidad de éxito. En particular, para el equipo que implementa la Encuesta Social Europea en España, parece que para conseguir una mayor efectividad la estrategia más rentable probablemente consista en:

- Concentrar los esfuerzos de conversión en hombres mayores de 40 años, que viven en pisos o apartamentos en zonas urbanas, principalmente en grandes ciudades 0 suburbios, en barrios con edificios en buen estado físico.

- Concentrar los esfuerzos de localización en hombres menores de 40 años, que vivan en pisos o apartamentos en pequeñas ciudades, en barrios con edificios en buen estado físico.

Tabla 19.

Características que incrementan la probabilidad de pertenecer al grupo de convertidos y al grupo de localizados

(respecto a la probabilidad de acceder directamente a la entrevista)

\begin{tabular}{ll}
\hline Convertidos & Localizados \\
\hline Hombre & Hombre \\
Mayor (a partir de 40 años) & Joven (de menos de 30 años) \\
Grandes ciudades o suburbios & Pequeñas ciudades \\
Pisos o apartamentos & Pisos o apartamentos \\
Edificios en buen o muy buen estado & Edificios en buen o muy buen estado \\
\hline
\end{tabular}




\section{CONCLUSIONES}

Aumentar la tasa de respuesta de cualquier encuesta debe ser un objetivo prioritario para obtener datos de calidad. Los problemas que genera una tasa de respuesta baja son realmente insuperables desde el punto de vista técnico. El rechazo a participar en encuestas y el fracaso en la localización son dos problemas básicos que afectan de un modo importante al objetivo de contar con una tasa de respuesta aceptable. Por ello uno de los requerimientos técnicos más importante de la ESE es alcanzar al menos una tasa de un 70 por ciento.

El esfuerzo realizado por el equipo español de la ESE para mejorar la tasa de respuesta de las sucesivas olas de esta encuesta, y en concreto de la tercera ola, ha obtenido unos buenos resultados, alcanzando en la última ola una tasa de respuesta mucho más próxima al objetivo del $70 \%$ fijado por el protocolo de la ESE en Europa. Los esfuerzos encaminados a un mejor entrenamiento de los entrevistadores, proponer mecanismos de incentivos monetarios para los mismos y para la propia empresa, desarrollar estrategias de mejor acceso a los entrevistados como cartas previas de contacto, o proporcionar números 900 a los entrevistados para que comprueben la existencia de dicha encuesta, buscar horas de contacto y entrevista fuera de los horarios de trabajo habituales, determinar y alcanzar un número determinado de contactos antes de desechar la unidad y otras más que se han detallado anteriormente, parecen obtener resultados significativos. En este trabajo se muestra que tales esfuerzos, encaminados a aumentar la tasa de respuesta, tienen consecuencias visibles y mensurables, algo que se ha podido comprobar mediante el llamado formulario de contacto, que también forma parte de la ESE y que incluye información básica sobre el trabajo de campo y el desarrollo de la entrevista.

Por otro lado, los datos obtenidos con este mismo formulario nos han permitido construir una tipología según el decurso de los contactos y entrevistas de cada una de las unidades iniciales de la muestra, de modo que hemos clasificado a los individuos de nuestra muestra en personas no contactadas, contactadas con posterioridad a unos inicios infructuosos, aquéllas que rechazaron inicialmente pero que se logró entrevistar posteriormente, aquéllas que permanecieron en el rechazo a la entrevista y, finalmente aquellas personas que aceptaron la entrevista desde un primer momento. Esta clasificación, junto con los datos adicionales de un conjunto de variables recogidos en dicho formulario de contacto, nos ha permitido poder identificar claramente un conjunto de variables que definen el perfil de cada uno de estos grupos de individuos recogidos inicialmente en nuestra muestra.

Toda esta información no sólo muestra los efectos de determinadas estrategias de trabajo de campo, sino que nos proporciona información básica para futuros trabajos que quieran alcanzar tasas de respuesta superiores en las encuestas que se vayan a realizar en España y que aspiren a tener tasas de respuesta mejores que garanticen unos datos de calidad. 


\section{REFERENCIAS BIBLIOGRÁFICAS}

Bethlehem, J. G. 2002. "Weighting nonresponse adjustments based on auxiliary information." Pp. 265287 en Survey nonresponse. Grooves, R. M., D. A. Dillman, J. L. Eltinge y R. J. A. Little. Nueva York: Wiley.

Bradburn, N. M. 1985. "Response effects." Pp. 289-328 en Rossi, P. H., J. D. Wright y A. B. Anderson (coords.), Handbook of survey research. San Diego: Academic Press.

Briemer, P. y L. E. Lyberg. 2003. Introduction to Survey Quality. Hoboken: John Wiley and sons.

Cuxart, A y C. Riba. 2009. "Mejorando a partir de la experiencia: la implementación de la tercera ola de la ESE en España." Revista Española de Investigaciones Sociológicas 125: 147-168.

Cuxart, A. y C. Riba. 2005. "Aspectos metodológicos de la Encuesta Social Europea." Pp. 21-40 en Torcal, M., S. Pérez Nievas y L. Morales (coords.), España: sociedad y política en perspectiva comparada. Un análisis de la primera ola de la Encuesta Social Europea. Valencia: Tirant lo Blanch.

Fowler, F. 2002. Survey Research Methods. Thousand Oaks: Sage.

Grooves, R. 1989. Survey Errors and Survey Cost. Nueva York: Wiley and Sons.

Kish, L. 1965. Survey Sampling. Nueva York: Wiley and Sons.

Marker, D. 2002. "Improving Quality of Surveys", trabajo presentado en la conferencia Improving Quality of Surveys, Copenague. Agosto. Consultado el 1 de diciembre de 2008 (http://www.icis.dk/ICIS_ papers/Keynote2_0_2.pdf)

Philippens, M. y J. Billiet. 2004. "Monitoring and evaluating nonresponse issues and fieldwork efforts in the European Social Survey", trabajo presentado en la European Conference on quality and methodology in official statistics, Mainz, Germany. Mayo (http://ess,nsd,uib,no/index,jsp?year=200 3\&module=documentation\&country, en ESS1 - Data quality assessment). Consultado 1-12-2008.

Riba, C. y A. Cuxart. 2003. "Construyendo las bases para una comparación fiable: la Encuesta Social Europea 2002 en España." Revista Española de Ciencia Política 8: 165-185.

Saris, W. E. and I. Gallhofer. 2007. Design Evaluation and Analysis of Questionnaires for Survey Research. New Jersey: John Wiley and Sons.

Saris, W. E. y A. Münnich. 1995. The Multitrait-Multimethod Approach to Evaluate Measurement Instruments. Budapest: Eötvos University Press.

Saris, W. E., A. Satorra y G. Coenders. 2004. "A new approach to evaluating the quality of measurement instruments: the split-ballot MTMM design." Sociological Methodology 34: 311-347.

Stoop, I. A. L. 2005. The Hunt for the Last Respondent. La Haya: Social and Cultural Planning Office of the Netherlands.

Sudnam, S. 1983. "Applied sampling." Pp. 145-194 en Rossi, P. H., J. D. Wright y A. B. Anderson (coords.), Handbook of survey research. San Diego: Academic Press. 
Torcal, M., L. Morales y C. Riba. 2006. "Supervisión y control de calidad del trabajo de campo de la Encuesta Social Europea en España: Evaluación y resultados." Metodología de Encuestas 7: $75-97$

CLARA RIBA es Doctora en Ciencias Políticas y de la Administración y Diplomada en Recopilación y Análisis de Datos en Ciencias Sociales. Ha trabajado en temas relacionados con comportamiento político, gestión educativa y evaluación de políticas públicas y es miembro del equipo que implementa la Encuesta Social Europea en España.

MARIANO TORCAL (Madrid, 1962) es Catedrático de Ciencia Política de la Universitat Pompeu Fabra y Coordinador Nacional de la European Social Survey (ESS) en España. Libros recientes publicados son Political Disaffection in Contemporary Democracies. Social Capital, Institutions and Politics (Londres: Routledge, 2006, coeditado con José Ramón Montero); Ciudadanos, asociaciones y participación en España (Madrid: CIS, 2006, coeditado con José Ramón Montero y Joan Font) y Las Elecciones Generales del 2004 (Madrid: CIS, 2007, coeditado con José Ramón Montero e Ignacio Lago).

LAURA MORALES es investigadora en el Instituto de Cambio Social de la Universidad de Manchester y Doctora en Ciencias Políticas por la Universidad Autónoma de Madrid. Ha publicado diversos artículos y capítulos de libro sobre participación política y asociacionismo. Entre 2001 y 2007 formó parte del equipo español de la Encuesta Social Europea.

RECIBIDO: $17 / 12 / 2008$

ACEPTADO: $21 / 05 / 2009$ 